\title{
Space-Time Diffeomorphisms in Noncommutative Gauge Theories*
}

\author{
Marcos ROSENBAUM, J. David VERGARA and L. Román JUAREZ \\ Instituto de Ciencias Nucleares, Universidad Nacional Autónoma de México, \\ A. Postal 70-543, México D.F., México \\ E-mail: mrosen@nucleares.unam.mx,vergara@nucleares.unam.mx, \\ roman.juarez@nucleares.unam.mx
}

Received April 11, 2008, in final form June 25, 2008; Published online July 16, 2008

Original article is available at http://www.emis.de/journals/SIGMA/2008/055/

\begin{abstract}
In previous work [Rosenbaum M. et al., J. Phys. A: Math. Theor. 40 (2007), 10367-10382] we have shown how for canonical parametrized field theories, where spacetime is placed on the same footing as the other fields in the theory, the representation of space-time diffeomorphisms provides a very convenient scheme for analyzing the induced twisted deformation of these diffeomorphisms, as a result of the space-time noncommutativity. However, for gauge field theories (and of course also for canonical geometrodynamics) where the Poisson brackets of the constraints explicitely depend on the embedding variables, this Poisson algebra cannot be connected directly with a representation of the complete Lie algebra of space-time diffeomorphisms, because not all the field variables turn out to have a dynamical character [Isham C.J., Kuchař K.V., Ann. Physics 164 (1985), 288-315, 316333]. Nonetheless, such an homomorphic mapping can be recuperated by first modifying the original action and then adding additional constraints in the formalism in order to retrieve the original theory, as shown by Kuchař and Stone for the case of the parametrized Maxwell field in [Kuchař K.V., Stone S.L., Classical Quantum Gravity 4 (1987), 319-328]. Making use of a combination of all of these ideas, we are therefore able to apply our canonical reparametrization approach in order to derive the deformed Lie algebra of the noncommutative space-time diffeomorphisms as well as to consider how gauge transformations act on the twisted algebras of gauge and particle fields. Thus, hopefully, adding clarification on some outstanding issues in the literature concerning the symmetries for gauge theories in noncommutative space-times.
\end{abstract}

Key words: noncommutativity; diffeomorphisms; gauge theories

2000 Mathematics Subject Classification: 70S10; 70S05; 81T75

\section{Introduction}

Within the context of quantum field theory, a considerable amount of work has been done recently dealing with quantum field theories in noncommutative space-times (NCQFT). One of the most relevant issues in this area is related to the symmetries under which these noncommutative systems are invariant. The most recent contention being that NCQFT are invariant under global "twisted symmetries" (see, e.g., [5]). This criterion has been extended to the case of the twisting of local symmetries, such as diffeomorphisms [6], and this has been used to propose some noncommutative theories of gravity $[6,7,8]$. Another possible extension of this idea is to consider the construction of noncommutative gauges theories with an arbitrary gauge group $[9,10]$. Regarding this latter line of research there is, however, some level of controversy as to whether it is possible to construct twisted gauge symmetries $[11,12,13]$. In this

${ }^{\star}$ This paper is a contribution to the Special Issue on Deformation Quantization. The full collection is available at http://www.emis.de/journals/SIGMA/Deformation_Quantization.html 
work we address this issue from the point of view of canonically reparametrized field theories. It is known indeed that for the case of field theories with no internal symmetries, it is possible to establish, within the framework of the canonical parametrization, an anti-homorphism between the Poisson algebra of the constraints on the phase space of the system and the algebra of space-time diffeomorphisms [2,3]. Using this anti-homomorphism we were able in [1] to show how the deformations of the algebra of constraints, resulting from space-time noncommutativity at the level of the quantum mechanical mini-superspace, are reflected on the twisting of the algebra of the fields as well as in the Lie algebra of the twisted diffeomorphisms and in the ensuing twisting of the original symmetry group of the theory. However, as it has also been noted by Isham and Kuchař in [2,3], for the case of gauge theories, there are some difficulties in representing space-time diffeomorphisms by an anti-homomorphic mapping into the Poisson algebra of the dynamical variables on the extended phase space of the canonically reparametrized theory, due to the fact that because of the additional internal symmetries some components of the field loose their dynamical character and appear as Lagrange multipliers in the formalism.

Nonetheless, as it was exemplified in [4] for the case of the parametrized Maxwell field, such difficulties can be circumvented and the desired mapping made possible by adding some terms to the original action and some additional constraints in order to recover the original features of the theory.

Making therefore use of the specific results derived by Kuchař and Stone in [4] for the parametrized Maxwell field and the re-established mapping between the space-time diffeomorphisms and the Poisson algebra of the modified theory, together with our previous results in [14] - whereby noncommutativity in field theory, manifested as the twisting of the algebra of fields, has a dynamical origin in the quantum mechanical mini-superspace which, for flat Minkowski space-time, is related to an extended Weyl-Heisenberg group - and including this results into a generalized symplectic structure of the parametrized field theory [1], we show here how our approach can be extended to gauge field theories thus allowing us to derive the deformed Lie algebra of the noncommutative space-time diffeomorphisms, as well as to consider how the gauge transformations act on the twisted algebras of gauge and particle fields. Hopefully this approach will help shed some additional univocal light on the above mentioned controversy.

The paper is organized as follows: In Section 2 we review the essential aspects of the construction of canonical parametrized field theories and representations of space-time diffeomorphisms, following $[2,3,15,16]$. In Section 3 we show how the formalism can be extended to the case of parametrized gauge field theories by making use of the ideas formulated in [4] in the context of Maxwell's electrodynamics. Section 4 summarizes in the language of Principal Fiber Bundles (PFB) some of the basic aspects of the theory of gauge transformations which will be needed in the later part of the work. In Section 5 we combine the results of the previous sections in order to extend the formalism to the noncommutative space-time case, by deforming the symplectic structure of the theory to account for the noncommutativity of the space-time embedding coordinates.

We thus derive a deformed algebra of constraints in terms of Dirac-brackets which functionally satisfy the same Dirac relations as those for the commutative case and can therefore be related anti-homomorphically to a Lie algebra of generators of twisted space-time diffeomorphisms. On the basis of these results we further show how, in order to preserve the consistency of the algebra of constraints, the Lie algebra of these generators of space-time diffeomorphisms and those of the gauge symmetry are in turn related.

Finally by extending the algebra of twisted diffeomorphisms to its universal covering, it was given an additional Hopf structure which allowed us to relate the twisting of symmetry of the theory to the Drinfeld twist. 


\section{Space-time diffeomorphisms in parametrized gauge theories}

As it is well known, see e.g. [2, 3], for Poincaré invariant field theory on a flat Minkowskian background, each generator of the Poincaré Lie algebra, represented by a dynamical variable on the phase-space of the field, is mapped homomorphically into the Poisson bracket algebra of these dynamical variables.

On a curved space-time background field theories are not Poincaré invariant but, by a parametrization consisting of extending the phase-space by adjoining to it the embedding variables, they can be made invariant under arbitrary space-time diffeomorphisms [17, 18]. Hence spacetime parameters are raised to the level of fields on the same footing as the original fields in the theory. Moreover, in this case it can also be shown [2] that:

a) An anti-homomorphic mapping can be established from the Poisson algebra of dynamical variables on the extended phase-space and the Lie algebra $£ \operatorname{diff} \mathcal{M}$ of arbitrary space-time diffeomorphisms. Thus,

$$
\left\{H_{\tau}[\xi], H_{\tau}[\eta]\right\}=-H_{\tau}\left[£_{\xi} \eta\right],
$$

where $\xi, \eta \in £ \operatorname{diff} \mathcal{M}$ are two complete space-time Hamiltonian vector fields on $\mathcal{M}, H_{\tau}[\xi]:=$ $\int_{\Sigma} d \boldsymbol{\sigma} \xi^{\alpha} \mathcal{H}_{\alpha}$, and $\mathcal{H}_{\alpha}$ are the constraints (supermomenta and superHamiltonian) of the theory, satisfying the Dirac vanishing Poisson bracket algebra

$$
\left\{\mathcal{H}_{\alpha}(\boldsymbol{\sigma}), \mathcal{H}_{\beta}\left(\boldsymbol{\sigma}^{\prime}\right)\right\} \simeq 0 .
$$

b) The Poisson brackets of the canonical variables representing the $£ \operatorname{diff} \mathcal{M}$ correctly induce the displacements of embeddings accompanied by the evolution of the field variables, predicted by the field equations.

For the prescribed pseudo-Riemannian background $\mathcal{M}$, equipped with coordinates $X^{\alpha}$, reparametrization involves a foliation $\Sigma \times \mathbb{R}$ of this space-time, where $\mathbb{R}$ is a temporal direction labeled by a parameter $\tau$ and $\Sigma$ is a space-like hypersurface of constant $\tau$, equipped with coordinates $\sigma^{a}$ $(a=1,2,3)$, and embedded in the space-time 4-manifold by means of the mapping

$$
X^{\alpha}=X^{\alpha}\left(\sigma^{a}\right) .
$$

This hypersurface is assumed to be spacelike with respect to the metric $g_{\alpha \beta}$ on $\mathcal{M}$, with signature $(-,+,+,+)$.

Let now the embedding functionals $X^{\alpha}{ }_{a}(\boldsymbol{\sigma}, X):=\frac{\partial X^{\alpha}(\boldsymbol{\sigma})}{\partial \sigma^{a}}$ and $n^{\alpha}(\boldsymbol{\sigma}, X)$, defined by

$$
g_{\alpha \beta} X^{\alpha}{ }_{a} n^{\beta}=0, \quad \text { and } \quad g_{\alpha \beta} n^{\alpha} n^{\beta}=-1,
$$

be an anholonomic basis consisting of tangent vectors to the hypersurface and unit normal, respectively.

We can therefore write the constraints $\mathcal{H}_{\alpha}$ as

$$
\mathcal{H}_{\alpha}=-\mathcal{H}_{\perp} n_{\alpha}+\mathcal{H}_{a} X_{\alpha}{ }^{a},
$$

where $\mathcal{H}_{\perp}$ and $\mathcal{H}_{a}$ are the super-Hamiltonian and super-momenta constraints, respectively. Using this decomposition the Dirac relations (2.1) can be written equivalently as

$$
\begin{aligned}
& \left\{\mathcal{H}_{\perp}(\boldsymbol{\sigma}), \mathcal{H}_{\perp}\left(\boldsymbol{\sigma}^{\prime}\right)\right\}=\sum_{a=1}^{3} \gamma^{a b} \mathcal{H}_{b}(\boldsymbol{\sigma}) \partial_{\sigma^{a}} \delta\left(\boldsymbol{\sigma}-\boldsymbol{\sigma}^{\prime}\right)-\left(\boldsymbol{\sigma} \leftrightarrow \boldsymbol{\sigma}^{\prime}\right), \\
& \left\{\mathcal{H}_{a}(\boldsymbol{\sigma}), \mathcal{H}_{b}\left(\boldsymbol{\sigma}^{\prime}\right)\right\}=\mathcal{H}_{b}(\boldsymbol{\sigma}) \partial_{\sigma^{a}} \delta\left(\boldsymbol{\sigma}-\boldsymbol{\sigma}^{\prime}\right)+\mathcal{H}_{a}\left(\boldsymbol{\sigma}^{\prime}\right) \partial_{\sigma^{b}} \delta\left(\boldsymbol{\sigma}-\boldsymbol{\sigma}^{\prime}\right), \\
& \left\{\mathcal{H}_{a}(\boldsymbol{\sigma}), \mathcal{H}_{\perp}\left(\boldsymbol{\sigma}^{\prime}\right)\right\}=\mathcal{H}_{\perp}(\boldsymbol{\sigma}) \partial_{\sigma^{a}} \delta\left(\boldsymbol{\sigma}-\boldsymbol{\sigma}^{\prime}\right),
\end{aligned}
$$


where $\gamma^{a b}$ is the inverse of the spatial metric

$$
\gamma_{a b}(\boldsymbol{\sigma}, X):=g_{\alpha \beta}(X(\boldsymbol{\sigma})) X_{a}^{\alpha} X_{b}^{\beta} .
$$

Also, as a consequence of the antihomomorphism between the Poisson algebra of the constraints and $£ \operatorname{diff} \mathcal{M}$ we can write

$$
H_{\tau}[\xi] \leadsto \hat{H}_{\tau}[\xi] \equiv \delta_{\xi}=\left.\xi^{\alpha}(X(\tau, \boldsymbol{\sigma})) \frac{\partial}{\partial X^{\alpha}}\right|_{X(\tau, \boldsymbol{\sigma})} .
$$

Indeed, since $[\eta, \rho]=£_{\eta} \rho$ we have

$$
\begin{aligned}
{\left[\delta_{\eta}, \delta_{\rho}\right] \phi } & =\delta_{£_{\eta} \rho} \phi=\hat{H}_{\tau}\left[£_{\eta} \rho\right] \triangleright \phi \cong\left\{\phi, H_{\tau}\left[£_{\eta} \rho\right]\right\} \\
& =\hat{H}_{\tau}[\eta] \triangleright\left[\hat{H}_{\tau}[\rho] \triangleright \phi\right]-\hat{H}_{\tau}[\rho] \triangleright\left[\hat{H}_{\tau}[\eta] \triangleright \phi\right] \\
& \cong\left\{\left\{\phi, H_{\tau}[\rho]\right\}, H_{\tau}[\eta]\right\}-\left\{\left\{\phi, H_{\tau}[\eta]\right\}, H_{\tau}[\rho]\right\}=-\left\{\phi,\left\{H_{\tau}[\eta], H_{\tau}[\rho]\right\}\right\}
\end{aligned}
$$

after resorting to the Jacobi identity and where $\phi$ is some field function in the theory.

Making use of this antihomomorphism as well as of the dynamical origin of $\star$-noncommutativity in field theory from quantum mechanics exhibited in [14], we have considered in [1] the extension of the reparametrization formalism and the canonical representation of space-time diffeomorphisms to the study of field theories on noncommutative space-times. More specifically, in that paper we discussed the particular case of a Poincaré invariant scalar field immersed on a flat Minkowskian background, and showed that the deformation of the algebra of constraints due to the incorporation of a symplectic structure in the theory originated the Drinfeld twisting of that isometry. However, although the formalism developed there can be extended straightforwardly to any field theory with no internal symmetries, for the case of parametrized gauge theories some additional complications arise, as pointed out in [3] and [4], due to the fact that the components of the gauge field perpendicular to the embedding are not dynamical but play instead the role of Lagrange multipliers which are not elements of the extended phase space and therefore can not be turned into dynamical variables by canonical transformations. To do so, and recover the anti-homomorphism between the algebra of space-time diffeomorphisms and

the Poisson algebra of constraints it is necessary to impose additional Gaussian conditions. The simplest case where such a procedure can be exhibited is the parametrized electromagnetic field. This has been very clearly elaborated in [4], so we shall only review those aspects of that work needed for our presentation.

\section{Parametrized Maxwell field and canonical representation of space-time diffeomorphisms}

Consider a source-free Maxwell field in a prescribed pseudo-Riemannian space-time represented by the action

$$
S=-\frac{1}{4} \int d^{4} X \sqrt{-g} g^{\mu \nu} g^{\alpha \beta} F_{\mu \alpha} F_{\nu \beta},
$$

where $F_{\mu \alpha}=A_{[\mu, \alpha]}:=A_{\mu, \alpha}-A_{\alpha, \mu}$. In the canonical treatment of the evolution of a field one assumes it to be defined on a space-like 3-hypersurface $\Sigma$, equipped with coordinates $\sigma$, which is embedded in the space-time manifold $\mathcal{M}$ by the mapping

$$
X^{\mu}:(\boldsymbol{\sigma})=X^{\mu}\left(\sigma^{a}\right), \quad a=1,2,3 .
$$

By adjoining the embedding variables to the phase space of the field results in a parametrized field theory where the space-time coordinates have been promoted to the rank of fields. In 
terms of the space-time coordinates $\sigma^{\alpha}=(\tau, \boldsymbol{\sigma})$ determined by the foliation $\mathcal{M}=\mathbb{R} \times \Sigma$, the action (3.1) becomes

$$
S=-\frac{1}{4} \int d \tau d^{3} \sigma \sqrt{-\bar{g}} \bar{g}^{\mu \nu} \bar{g}^{\alpha \beta} F_{\mu \alpha} F_{\nu \beta}, \quad t \in \mathbb{R},
$$

with the inverse metric $\bar{g}^{\alpha \beta}$ given by

$$
\bar{g}^{\alpha \beta}=\frac{\partial \sigma^{\alpha}}{\partial X^{\mu}} \frac{\partial \sigma^{\beta}}{\partial X_{\mu}},
$$

which can be therefore seen as a function of the coordinate fields. In (3.2) $\bar{g}:=\operatorname{det}\left(\bar{g}_{\mu \nu}\right)$ where $\sqrt{-\bar{g}}=J$ is the Jacobian of the transformation.

In order to carry out the Hamiltonian analysis of the action (3.2), we define in similar way to $(2.2)$ the tangent vectors to $\Sigma, X_{a}^{\alpha}$ and the unit normal $n^{\alpha}=-\left(-\bar{g}^{00}\right)^{-\frac{1}{2}} \bar{g}^{0 \rho} \frac{\partial X^{\alpha}}{\partial \sigma^{\rho}}$. We thus arrive at

$$
S\left[X^{\mu}, P_{\mu}, A_{a}, \pi^{a}, A_{\perp}\right]=\int d \tau d^{3} \sigma\left(P_{\alpha} \dot{X}^{\alpha}+\pi^{a} \dot{A}_{a}-N \Phi_{0}-N^{a} \Phi_{a}-M G\right),
$$

where $N$ and $N^{a}$ are the lapse and shift components of the deformation vector $N^{\alpha}:=\partial X^{\alpha} / \partial \tau$, $M=N A_{\perp}-N^{a} A_{a}$, and $A_{a}:=X_{a}^{\alpha} A_{\alpha}, A_{\perp}:=-n^{\beta} A_{\beta}$ are the tangent and normal projections of the gauge potential. The constraints $\Phi_{0}, \Phi_{a}$ and $G$ in (3.3) are defined by:

$$
\begin{aligned}
& \Phi_{0}=P_{\alpha} n^{\alpha}+\frac{1}{2} \gamma^{-1 / 2} \gamma_{a b} \pi^{a} \pi^{b}+\frac{1}{4} \gamma^{1 / 2} \gamma^{a c} \gamma^{b d} F_{a b} F_{c d}, \\
& \Phi_{a}=P_{\alpha} X_{, a}^{\alpha}+F_{a b} \pi^{b}, \quad G=\pi_{, a}^{a},
\end{aligned}
$$

where $\gamma_{a b}, \gamma$ are the metric components on $\Sigma$ and their determinant, respectively. These constraints satisfy the relations:

$$
\begin{aligned}
& \left\{\Phi_{0}(\boldsymbol{\sigma})+A_{\perp}(\boldsymbol{\sigma}) G(\boldsymbol{\sigma}), \Phi_{0}\left(\boldsymbol{\sigma}^{\prime}\right)+A_{\perp}\left(\boldsymbol{\sigma}^{\prime}\right) G\left(\boldsymbol{\sigma}^{\prime}\right)\right\}=\left[\gamma^{a b}(\boldsymbol{\sigma}) \Phi_{b}(\boldsymbol{\sigma})+\gamma^{a b}\left(\boldsymbol{\sigma}^{\prime}\right) \Phi_{b}\left(\boldsymbol{\sigma}^{\prime}\right)\right] \delta_{, a}\left(\boldsymbol{\sigma}, \boldsymbol{\sigma}^{\prime}\right), \\
& \left\{\Phi_{a}(\boldsymbol{\sigma})-A_{a}(\boldsymbol{\sigma}) G(\boldsymbol{\sigma}), \Phi_{b}\left(\boldsymbol{\sigma}^{\prime}\right)-A_{b}\left(\boldsymbol{\sigma}^{\prime}\right) G\left(\boldsymbol{\sigma}^{\prime}\right)\right\} \\
& \quad=\left(\Phi_{b}(\boldsymbol{\sigma})-A_{b}(\boldsymbol{\sigma}) G(\boldsymbol{\sigma})\right) \delta_{, a}\left(\boldsymbol{\sigma}, \boldsymbol{\sigma}^{\prime}\right)+\left(\Phi_{a}\left(\boldsymbol{\sigma}^{\prime}\right)-A_{a}\left(\boldsymbol{\sigma}^{\prime}\right) G\left(\boldsymbol{\sigma}^{\prime}\right)\right) \delta_{, b}\left(\boldsymbol{\sigma}, \boldsymbol{\sigma}^{\prime}\right), \\
& \left\{\Phi_{a}(\boldsymbol{\sigma})-A_{a}(\boldsymbol{\sigma}) G(\boldsymbol{\sigma}), \Phi_{0}\left(\boldsymbol{\sigma}^{\prime}\right)+A_{\perp}\left(\boldsymbol{\sigma}^{\prime}\right) G\left(\boldsymbol{\sigma}^{\prime}\right)\right\}=\left(\Phi_{0}(\boldsymbol{\sigma})+A_{\perp}(\boldsymbol{\sigma}) G(\boldsymbol{\sigma})\right) \delta_{, a}\left(\boldsymbol{\sigma}, \boldsymbol{\sigma}^{\prime}\right), \\
& \left\{\Phi_{0}(\boldsymbol{\sigma}), G\left(\boldsymbol{\sigma}^{\prime}\right)\right\}=0, \quad\left\{\Phi_{a}(\boldsymbol{\sigma}), G\left(\boldsymbol{\sigma}^{\prime}\right)\right\}=0 .
\end{aligned}
$$

From here we see that the Gauss constraint $G$ is needed to achieve the closure of the algebra of the super-Hamiltonian and super-momenta constraints, $\Phi_{0}, \Phi_{a}$, under the Poisson-brackets. However, because of the gauge invariance implied by the Gauss constraint $G \approx 0$, the scalar potential $A_{\perp}$ occurs in (3.5) not as a dynamical variable but as a Lagrange multiplier. The end result of this mixing of constraints and consequent foliation dependence of the space-time action in gauge theories, is that the super-Hamiltonian,

$$
n^{\alpha} \mathcal{H}_{\alpha}=\mathcal{H}_{\perp}:=\Phi_{0}(\boldsymbol{\sigma})+A_{\perp}(\boldsymbol{\sigma}) G(\boldsymbol{\sigma}),
$$

and the supermomenta,

$$
X_{a}^{\alpha} \mathcal{H}_{\alpha}=\mathcal{H}_{a}:=\Phi_{a}(\boldsymbol{\sigma})-A_{a}(\boldsymbol{\sigma}) G(\boldsymbol{\sigma})
$$

constraints do not satisfy the Dirac closure relations $(2.1)\left(\left\{\mathcal{H}_{\alpha}(\boldsymbol{\sigma}), \mathcal{H}_{\beta}\left(\boldsymbol{\sigma}^{\prime}\right)\right\} \simeq 0\right)$, so we do not have a direct homomorphic map from the Poisson brackets algebra of constraints into the Lie algebra of space-time diffeomorphisms for such theories. Nonetheless, this difficulty can be 
circumvented by turning the scalar potential into a canonical momentum $\pi$ (via the relation $\pi=\sqrt{\gamma} A_{\perp}$ ) conjugate to a supplementary scalar field $\psi$ and prescribing their dynamics by imposing the Lorentz gauge condition. The new super-Hamiltonian and super-momenta

$$
{ }^{*} \mathcal{H}_{\perp}:=\mathcal{H}_{\perp}-\sqrt{\gamma} \gamma^{a b} \psi_{, a} A_{b}, \quad{ }^{*} \mathcal{H}_{a}:=\mathcal{H}_{a}+\pi \psi_{, a},
$$

of the modified theory satisfy the Dirac closure relations, and the mapping $\xi \rightarrow{ }^{*} H_{\tau}[\xi]=$ $\int_{\Sigma} d \boldsymbol{\sigma}^{\prime} \xi^{\alpha}\left(X\left(\boldsymbol{\sigma}^{\prime}\right)\right)^{*} \mathcal{H}_{\alpha}$ results in the desired anti-homomorphism:

$$
\left\{{ }^{*} H_{\tau}[\xi],{ }^{*} H_{\tau}[\rho]\right\}=-{ }^{*} H_{\tau}\left[£_{\xi} \rho\right]
$$

from the Lie algebra $£ \operatorname{diff} \mathcal{M} \ni \xi, \rho$ into the Poisson algebra of the constraints on the extended phase space $A_{a}, \pi^{a}, \psi, \pi, X^{\alpha}, P_{\alpha}$ of the modified electrodynamics with the space-time action:

$$
S(\phi, \psi)=\int_{\mathcal{M}} d^{4} X \sqrt{-g}\left(-\frac{1}{4} F^{\alpha \beta} F_{\alpha \beta}+\psi{ }_{, \alpha} g^{\alpha \beta} A_{\beta}\right) .
$$

Note however that in order to recover Maxwell's electrodynamics from the dynamically minimal modified action (3.8), one needs to impose the additional primary and secondary constraints

$$
C(\boldsymbol{\sigma}):=\psi(\boldsymbol{\sigma}) \approx 0, \quad G(\boldsymbol{\sigma}) \approx 0
$$

on the phase space data. In this way, the new algebra of constraints leading to vacuum electrodynamics from (3.8) is:

$$
\begin{aligned}
& \left\{{ }^{*} \mathcal{H}_{\perp}(\boldsymbol{\sigma}),{ }^{*} \mathcal{H}_{\perp}\left(\boldsymbol{\sigma}^{\prime}\right)\right\}=\gamma^{a b}(\boldsymbol{\sigma}){ }^{*} \mathcal{H}_{b}(\boldsymbol{\sigma}) \delta_{, a}\left(\boldsymbol{\sigma}, \boldsymbol{\sigma}^{\prime}\right)-\left(\boldsymbol{\sigma} \leftrightarrow \boldsymbol{\sigma}^{\prime}\right), \\
& \left\{{ }^{*} \mathcal{H}_{a}(\boldsymbol{\sigma}),{ }^{*} \mathcal{H}_{\perp}\left(\boldsymbol{\sigma}^{\prime}\right)\right\}={ }^{*} \mathcal{H}_{\perp}(\boldsymbol{\sigma}) \delta_{, a}\left(\boldsymbol{\sigma}, \boldsymbol{\sigma}^{\prime}\right), \\
& \left\{{ }^{*} \mathcal{H}_{a}(\boldsymbol{\sigma}),{ }^{*} \mathcal{H}_{b}\left(\boldsymbol{\sigma}^{\prime}\right)\right\}={ }^{*} \mathcal{H}_{b}(\boldsymbol{\sigma}) \delta_{, a}\left(\boldsymbol{\sigma}, \boldsymbol{\sigma}^{\prime}\right)-\left(a \boldsymbol{\sigma} \leftrightarrow b \boldsymbol{\sigma}^{\prime}\right), \\
& \left\{C(\boldsymbol{\sigma}),{ }^{*} \mathcal{H}_{\perp}\left(\boldsymbol{\sigma}^{\prime}\right)\right\}=(\gamma){ }^{-\frac{1}{2}}(\boldsymbol{\sigma}) G(\boldsymbol{\sigma}) \delta\left(\boldsymbol{\sigma}, \boldsymbol{\sigma}^{\prime}\right), \\
& \left\{C(\boldsymbol{\sigma}),{ }^{*} \mathcal{H}_{a}\left(\boldsymbol{\sigma}^{\prime}\right)\right\}=C_{, a}(\boldsymbol{\sigma}) \delta\left(\boldsymbol{\sigma}, \boldsymbol{\sigma}^{\prime}\right), \\
& \left\{G(\boldsymbol{\sigma}),{ }^{*} \mathcal{H}_{\perp}\left(\boldsymbol{\sigma}^{\prime}\right)\right\}=\left((\gamma)^{\frac{1}{2}}(\boldsymbol{\sigma}) \gamma^{a b}(\boldsymbol{\sigma}) C_{, b}(\boldsymbol{\sigma}) \delta\left(\boldsymbol{\sigma}, \boldsymbol{\sigma}^{\prime}\right)\right)_{, a}, \\
& \left\{G(\boldsymbol{\sigma}),{ }^{*} \mathcal{H}_{a}\left(\boldsymbol{\sigma}^{\prime}\right)\right\}=\left(G(\boldsymbol{\sigma}) \delta\left(\boldsymbol{\sigma}, \boldsymbol{\sigma}^{\prime}\right)\right)_{, a} .
\end{aligned}
$$

This Poisson algebra implies that once the constraints (3.9) are imposed on the initial data they are preserved in the dynamical evolution generated by the total Hamiltonian associated with (3.8), so that if the derivations ${ }^{*} \hat{H}_{\tau}[\xi]:=\delta_{\xi}$ representing space-time diffeomorphisms start evolving a point of the extended phase space lying on the intersection of the constraint surfaces

$$
{ }^{*} \mathcal{H}_{\perp}(\boldsymbol{\sigma}) \approx 0 \approx \mathcal{H}_{a}(\boldsymbol{\sigma}) \quad \text { and } \quad C(\boldsymbol{\sigma}):=\psi(\boldsymbol{\sigma}) \approx 0 \approx G(\boldsymbol{\sigma}),
$$

the point will keep moving along this intersection.

In summary, we have seen that for canonically parametrized field theories with gauge symmetries in addition to space-time symmetries the Poisson algebra of the constraints does not agree with the Dirac relations and, therefore, cannot be directly interpreted as representing the Lie algebra of the generators of space-time diffeomorphisms. The reason being that because of the gauge invariance there are additional constraints in the theory which cause that not all the relevant variables are canonical variables. Following the arguments in [4] for the case of the electromagnetic field, we have seen that these difficulties can be circumvented by complementing the original action (3.1) with the addition of a term, containing the scalar field $\psi$, that enforces the Lorentz condition, so the modified action is given by (3.8). Varying this action with respect to the gauge potential $A_{\alpha}$ gives

$$
\frac{1}{2}\left(|\sqrt{g}|^{\frac{1}{2}} F^{\alpha \beta}\right)_{, \beta}=|\sqrt{g}|^{\frac{1}{2}} g^{\alpha \beta} \psi_{, \beta},
$$


which therefore implies that the modified action introduces a source term into the Maxwell equations, so the dynamical theory resulting from (3.8) is not the same as Maxwell's electrodynamics in vacuum. It is interesting to observe, parenthetically, that the charge source on the right of (3.11) is a real field and not a complex one as one would have expected. The dynamical character of $\psi$, however, is evident when differentiating this last equation with respect to $X^{\alpha}$ whereby, due to the vanishing of the left side, this field must satisfy the wave equation

$$
\psi_{, \alpha}{ }^{, \alpha}=0
$$

Consequently, in order to recover Maxwell's electrodynamics it was required that $\psi$ vanish or at least that it is a space-time constant. This was achieved by simply imposing additional constraints on the phase space data, given by (3.9), which (c.f. equation (4.10) in the next section) implies loosing the generator of gauge transformations. This procedure, and its generalization to the case of non-Abelian Yang-Mills fields then allows (still within the canonical group theoretical framework) to undo the projection and replace the Poisson bracket relations (3.5) by the genuine Lie algebra $£ \operatorname{diff} \mathcal{M}$ of space-time diffeomorphisms.

Note that even though the algebra in (3.10) involves derivatives of the constraints $G(\boldsymbol{\sigma})$ and $C(\boldsymbol{\sigma})$, these derivatives can be removed by simply using the identity

$$
J\left(\boldsymbol{\sigma}^{\prime}\right) \delta_{, a}\left(\boldsymbol{\sigma}, \boldsymbol{\sigma}^{\prime}\right)=J(\boldsymbol{\sigma}) \delta_{, a}\left(\boldsymbol{\sigma}, \boldsymbol{\sigma}^{\prime}\right)+J_{, a}(\boldsymbol{\sigma}) \delta\left(\boldsymbol{\sigma}, \boldsymbol{\sigma}^{\prime}\right),
$$

so the algebra does close, as it is to be expected from counting degrees of freedom.

As a consequence the elements ${ }^{*} H_{\tau}[\xi]$, together with $G_{\tau}[\bar{\alpha}]:=\int d \boldsymbol{\sigma} \bar{\alpha}(X(\boldsymbol{\sigma})) G(\boldsymbol{\sigma})$ and $C_{\tau}[\bar{\beta}]:=\int d \boldsymbol{\sigma} \bar{\beta}(X(\boldsymbol{\sigma})) C(\boldsymbol{\sigma})$, form a closed algebra under the Poisson brackets.

On the basis of the above discussion let us now derive explicit expressions for the generators of the Lie algebra of space-time diffeomorphisms associated with the anti-homomorphism (3.7) and investigate whether these Lie algebra can be extended with the smeared elements $G_{\tau}[\bar{\alpha}]$ and $C_{\tau}[\bar{\beta}]$ and, if so what would be the interpretation of such an extension. For this purpose let us first begin by deriving the Poisson bracket of the projection $A_{a}$ of the 4-vector potential field $A_{\alpha}$ on the hypersurface $\Sigma$ with ${ }^{*} H_{\tau}[\xi]$. Making use of (3.4) and (3.6) we get

$$
\begin{aligned}
\left\{A_{a}(\boldsymbol{\sigma}),{ }^{*} H_{\tau}[\xi]\right\} & =\int d \boldsymbol{\sigma}^{\prime}\left\{A_{a}(\boldsymbol{\sigma}),-\xi^{\alpha}\left(\boldsymbol{\sigma}^{\prime}\right) n_{\alpha}\left(\boldsymbol{\sigma}^{\prime}\right)^{*} \mathcal{H}_{\perp}+\xi^{\alpha} X_{\alpha}{ }^{b}\left(\boldsymbol{\sigma}^{\prime}\right){ }^{*} \mathcal{H}_{b}\right\} \\
& =-\xi^{\alpha} n_{\alpha} \gamma^{-\frac{1}{2}} \gamma_{a b} \pi^{b}+\left(\xi^{\alpha} A_{\alpha}\right)_{, a}+\xi^{\alpha} X_{\alpha}{ }^{b} F_{b a}=\left(£_{\boldsymbol{\xi}} A_{\beta}\right) X^{\beta}{ }_{a},
\end{aligned}
$$

after also making use of the expression

$$
\pi^{a}:=\frac{\delta \mathcal{L}}{\delta \dot{A}_{a}(\boldsymbol{\sigma})}=-\gamma^{\frac{1}{2}} \gamma^{a b} F_{\perp b},
$$

for the momentum canonical conjugate to $A_{a}$ (c.f. equation (3.10) in [4]). Now, since the right side of (3.12) represents another gauge vector potential on $\Sigma$, it clearly follows that

$$
\left\{\left\{A_{a}(\boldsymbol{\sigma}),{ }^{*} H_{\tau}[\xi]\right\},{ }^{*} H_{\tau}[\eta]\right\}=\left(£_{\boldsymbol{\eta}} £_{\boldsymbol{\xi}} A_{\beta}\right) X^{\beta}{ }_{a},
$$

and interchanging the symbols $\boldsymbol{\xi}, \boldsymbol{\eta}$ on the left side above, substracting and using the Jacobi identity, yields

$$
\left\{A_{a}(\boldsymbol{\sigma}),\left\{{ }^{*} H_{\tau}[\xi],{ }^{*} H_{\tau}[\eta]\right\}\right\}=-\left(£_{[\boldsymbol{\xi}, \boldsymbol{\eta}]} A_{\beta}\right) X^{\beta}{ }_{a} .
$$

We can therefore write the map

$$
\left\{A_{a}(\boldsymbol{\sigma}),{ }^{*} H_{\tau}[\xi]\right\} \leadsto{ }^{*} \hat{H}_{\tau}[\xi] \triangleright A_{a}(\boldsymbol{\sigma}),
$$


where

$$
\delta_{\boldsymbol{\xi}} \equiv{ }^{*} \hat{H}_{\tau}[\xi]:=\left(X^{\beta}{ }_{a} \circ £_{\boldsymbol{\xi}}\right)
$$

is a derivation operator which when acting on a 4 -vector potential $A_{\beta}$ it projects its Lie derivative onto the hypersurface $\Sigma$.

Consider next the Poisson bracket of the scalar field $\psi$ with ${ }^{*} H_{\tau}[\xi]$. Again, from (3.4) and (3.6) we get

$$
\left\{\psi(\boldsymbol{\sigma}),{ }^{*} H_{\tau}[\xi]\right\}=\left[\xi^{\alpha}\left(-n_{\alpha} \gamma^{-\frac{1}{2}} \pi^{a}{ }_{, a}+X_{\alpha}{ }^{a} \psi_{, a}\right)\right](\boldsymbol{\sigma}) .
$$

Similarly for the time evolution of $\psi$, derived from the total Hamiltonian, we obtain

$$
\dot{\psi}=\left\{\psi(\boldsymbol{\sigma}), \int d \boldsymbol{\sigma}^{\prime}\left(N^{*} \mathcal{H}_{\perp}\left(\boldsymbol{\sigma}^{\prime}\right)+N^{a *} \mathcal{H}_{a}\left(\boldsymbol{\sigma}^{\prime}\right)\right)\right\}=N \gamma^{-\frac{1}{2}} \pi^{a}{ }_{, a}+N^{a} \psi_{, a} .
$$

Moreover, since

$$
\dot{\psi}:=\frac{\partial X^{\alpha}}{\partial \tau} \psi_{, \alpha}=N^{\alpha} \psi_{, \alpha}=N^{\alpha}\left(n_{\alpha} \psi_{, \perp}+X_{\alpha}^{a} \psi_{, a}\right)=-N \psi_{, \perp}+N^{a} \psi_{, a}
$$

which when substituted into (3.15) implies that $\psi_{, \perp}=-\gamma^{-\frac{1}{2}} \pi^{a}, a$, and hence (from (3.14)) that

$$
\left\{\psi(\boldsymbol{\sigma}),{ }^{*} H_{\tau}[\xi]\right\}=\left(\xi^{\alpha} \psi_{, \alpha}\right)(\boldsymbol{\sigma})=£_{\boldsymbol{\xi}} \psi(\boldsymbol{\sigma}) .
$$

It clearly follows from this that

$$
\left\{\psi(\boldsymbol{\sigma}),\left\{{ }^{*} H_{\tau}[\xi],{ }^{*} H_{\tau}[\eta]\right\}\right\}=-£_{[\boldsymbol{\xi}, \boldsymbol{\eta}]} \psi(\boldsymbol{\sigma})
$$

so for the action of ${ }^{*} H_{\tau}[\xi]$ on scalar fields we can therefore also write the morphism (3.13), ${ }^{*} H_{\tau}[\xi] \leadsto \delta_{\boldsymbol{\xi}} \equiv{ }^{*} \hat{H}_{\tau}[\xi]:=\left(X^{\beta}{ }_{a} \circ £_{\boldsymbol{\xi}}\right)$, provided it is naturally understood that the surface projection $X^{\beta}{ }_{a}$ acts as an identity on scalars. It should be clear from the above analysis that these derivations $\delta_{\boldsymbol{\xi}}$, as defined in (3.13), are indeed full space-time diffeomorphisms.

Let us now turn to the elements $G(\boldsymbol{\sigma})$ and $C(\boldsymbol{\sigma})$ of the algebra of constraints (3.10). The Poisson algebra of the mapping $\bar{\alpha} \rightarrow G_{\tau[\bar{\alpha}]}=\int_{\Sigma} d \boldsymbol{\sigma}^{\prime} \bar{\alpha}\left(X\left(\boldsymbol{\sigma}^{\prime}\right)\right) G\left(\boldsymbol{\sigma}^{\prime}\right)$, with $A_{a}$ is

$$
\left\{A_{a}(\boldsymbol{\sigma}), G_{\tau}[\bar{\alpha}]\right\}=-\partial_{a} \bar{\alpha}
$$

and, making use of (3.12), we get

$$
\left\{\left\{A_{a}(\boldsymbol{\sigma}), G_{\tau}[\bar{\alpha}]\right\},{ }^{*} H_{\tau}[\xi]\right\}=-\left(£_{\boldsymbol{\xi}} \partial_{\beta} \bar{\alpha}\right) X^{\beta}{ }_{a} .
$$

Inverting the ordering of the constraints in the above brackets we also have

$$
\left\{\left\{A_{a}(\boldsymbol{\sigma}),{ }^{*} H_{\tau}[\xi]\right\}, G_{\tau}[\bar{\alpha}]\right\}=-\left\{\left(£_{\boldsymbol{\xi}} A_{\beta}\right) X^{\beta}{ }_{a}, G_{\tau}[\bar{\alpha}]\right\}=-\partial_{a}\left(\xi^{c} \partial_{c} \bar{\alpha}\right) .
$$

Subtracting now (3.17) from (3.18), and making use of the Jacobi identity on the left side of the equation, results in

$$
\left\{A_{a}(\boldsymbol{\sigma}),\left\{{ }^{*} H_{\tau}[\xi], G_{\tau}[\bar{\alpha}]\right\}\right\}=\partial_{a}\left(\xi^{\perp} \bar{\alpha}_{, \perp}\right) .
$$

Note that we could equally well have gotten this result by identifying $G_{\tau}[\bar{\alpha}]$ with a derivation through the map

$$
G_{\tau}[\bar{\alpha}] \leadsto \hat{G}_{\tau}[\bar{\alpha}]:=-\int_{\Sigma} d \boldsymbol{\sigma}^{\prime}\left(\partial_{b} \bar{\alpha}\right)\left(\boldsymbol{\sigma}^{\prime}\right) \frac{\delta}{\delta A_{b}\left(\boldsymbol{\sigma}^{\prime}\right)},
$$


which could be seen as resulting from integrating the smeared constraint by parts and identifying the canonical momentum $\pi^{b}$ with the functional derivative: $\pi^{b} \leadsto \hat{\pi}^{b}:=\frac{\delta}{\delta A_{b}\left(\boldsymbol{\sigma}^{\prime}\right)}$. Indeed, acting first on $A_{a}$ with the derivation operator (3.13) gives

$$
\begin{aligned}
\delta_{\boldsymbol{\xi}} \triangleright A_{a} & \equiv{ }^{*} \hat{H}_{\tau}[\xi] \triangleright A_{a}:=\left(X^{\beta}{ }_{a} \circ £_{\boldsymbol{\xi}}\right) A_{a} \\
& =-\xi^{\alpha} n_{\alpha} \gamma^{-\frac{1}{2}} \gamma_{a b} \pi^{b}+\left(\xi^{\alpha} n_{\alpha} A_{\perp}\right)_{, a}+\left(\xi^{c}\right)_{, a} A_{c}+\xi^{c} A_{a, c},
\end{aligned}
$$

which, when followed by the action of (3.20) results in

$$
\begin{aligned}
\hat{G}_{\tau}[\bar{\alpha}] \triangleright\left(\delta_{\boldsymbol{\xi}} \triangleright A_{a}\right) & =-\int_{\Sigma} d \boldsymbol{\sigma}^{\prime}\left(\partial_{b} \bar{\alpha}\right)\left(\boldsymbol{\sigma}^{\prime}\right) \frac{\delta}{\delta A_{b}\left(\boldsymbol{\sigma}^{\prime}\right)}\left(\left(\xi^{\alpha} n_{\alpha} A_{\perp}\right)_{, a}+\left(\xi^{c}\right)_{, a} A_{c}+\xi^{c} A_{a, c}\right)(\boldsymbol{\sigma}) \\
& =-\partial_{a}\left(\xi^{c} \partial_{c} \bar{\alpha}\right) .
\end{aligned}
$$

Alternating the order of the above derivations, a similar calculation gives

$$
\delta_{\boldsymbol{\xi}} \triangleright\left(\hat{G}_{\tau}[\bar{\alpha}] \triangleright A_{a}\right)=-\delta_{\boldsymbol{\xi}} \triangleright \partial_{a} \bar{\alpha}=£_{\boldsymbol{\xi}}\left(\partial_{\beta} \bar{\alpha}\right) X^{\beta}{ }_{a}=-\partial_{a}\left(\xi^{\gamma} \partial_{\gamma} \bar{\alpha}\right),
$$

and subtracting from this (3.21) yields

$$
\left[\delta_{\boldsymbol{\xi}}, \hat{G}_{\tau}[\bar{\alpha}]\right] \triangleright A_{a}=-\partial_{a}\left(\xi^{\perp} \partial_{\perp} \bar{\alpha}\right),
$$

which could be thought to imply an algebra homomorphism when compared with (3.19). Observe, however, that if we evaluate the Poisson bracket of ${ }^{*} H_{\tau}[\xi]$ and $G_{\tau}[\bar{\alpha}]$ directly from (3.4) and (3.6) we get

$$
\begin{aligned}
\left\{{ }^{*} H_{\tau}[\xi], G_{\tau}[\bar{\alpha}]\right\} & =\int_{\Sigma} d \boldsymbol{\sigma}\left(-\xi^{\perp} \bar{\alpha}_{, \perp} G+\xi^{\perp}\left(\partial_{a} \bar{\alpha}\right) \gamma^{\frac{1}{2}} \gamma^{a b} C_{, b}\right)(\boldsymbol{\sigma}) \\
& =-G_{\tau}\left[\xi^{\perp} \bar{\alpha}_{, \perp}\right]-C_{\tau}\left[\left(\xi^{\perp}\left(\partial_{a} \bar{\alpha}\right) \gamma^{\frac{1}{2}} \gamma^{a b}\right)_{, b}\right] .
\end{aligned}
$$

This result remains compatible with (3.19) because $C(\boldsymbol{\sigma})$ acts as a projector when operating on the gauge vector field $A_{a}$. But, because the right hand side of the equation contains a linear combination of the smeared constraints $G_{\tau}$ and $C_{\tau}$, there is no way that we could implement the mapping (3.20) to get an homomorphism between the Poisson bracket (3.22) and the Lie bracket $\left[\delta_{\boldsymbol{\xi}}, \hat{G}_{\tau}[\bar{\alpha}]\right]$, as may be easily seen in fact when calculating the later with (3.13) and (3.20).

Similarly, if we now consider the Poisson bracket of the map $\bar{\beta} \rightarrow C_{\tau}[\bar{\beta}]=\int_{\Sigma} d \boldsymbol{\sigma}^{\prime} \bar{\beta}\left(X\left(\boldsymbol{\sigma}^{\prime}\right)\right) C\left(\boldsymbol{\sigma}^{\prime}\right)$ with ${ }^{*} H_{\tau}[\xi]$ we find (again making use of (3.4) and (3.6)) that

$$
\begin{aligned}
\left\{C_{\tau}[\bar{\beta}],{ }^{*} H_{\tau}[\xi]\right\} & =\int_{\Sigma} d \boldsymbol{\sigma}\left[\xi^{\alpha} \bar{\beta}_{, \alpha} C+\xi^{\perp} \bar{\beta} \gamma^{-\frac{1}{2}} G+\xi^{a} \bar{\beta} C_{, a}\right](\boldsymbol{\sigma}) \\
& =C_{\tau}\left[\xi^{\alpha} \bar{\beta}_{, \alpha}-\left(\xi^{a} \bar{\beta}\right)_{, a}\right]+G_{\tau}\left[\xi^{\perp} \bar{\beta} \gamma^{-\frac{1}{2}}\right] .
\end{aligned}
$$

However, if we were to assume valid the derivation operator map $C_{\tau}[\bar{\beta}] \leadsto \hat{C}_{\tau}[\bar{\beta}]=\int_{\Sigma} d \boldsymbol{\sigma} \bar{\beta} \frac{\delta}{\delta \pi(\boldsymbol{\sigma})}$, it would then clearly follow that

$$
\left[\delta_{\boldsymbol{\xi}}, \hat{C}_{\tau}[\bar{\beta}]\right] \triangleright A_{a}=0 .
$$

This result immediately enters into conflict with (3.23), where such a morphism of algebras, involving $\hat{C}_{\tau}[\bar{\beta}]$ together with $(3.20)$, would yield

$$
\left\{A_{a},\left\{C_{\tau}[\bar{\beta}],{ }^{*} H_{\tau}[\xi]\right\}\right\} \leadsto\left[\delta_{\boldsymbol{\xi}}, \hat{C}_{\tau}[\bar{\beta}]\right] \triangleright A_{a}=-\partial_{a}\left(\xi^{\perp} \bar{\beta} \gamma^{-\frac{1}{2}}\right) .
$$

Consequently, the largest Lie algebra that we can associate with the Poisson algebra (3.10) is the one of space-time diffeomorphisms, given by the homomorphism implied by (3.7) and originating from the sub-algebra of the super-Hamiltonian and super-momenta described by the first 3 equations in (3.10). We shall return to this observation later on, as it is essential for our conclusions. First we need however to relate our results derived so far with some basic aspects of gauge theory as formulated from the point of view of principal fiber bundles. 


\section{Gauge transformations}

Recall (c.f. e.g. [21]) that a gauge transformation of a principal fiber bundle (PFB) $\pi: P \rightarrow \mathcal{M}$, with structure Lie group $\mathcal{G}$, is an automorphism $f: P \rightarrow P$ such that $f(p g)=f(p) g$ and the induced diffeomorphism $\bar{f}: \mathcal{M} \rightarrow \mathcal{M}$, defined by $\bar{f}(\pi(p))=\pi(f(p))$, is the identity map $\bar{f}=1_{\mathcal{M}}$ (i.e. $\pi(p)=\pi(f(p))$ ). Moreover, if we define $f: P \rightarrow P$ by $f(p)=p \zeta(p)$, where $\zeta$ is an element of the space $C(P, \mathcal{G})$ of all maps such that $\zeta(p g)=g^{-1} \cdot \zeta(p)=\operatorname{Ad}_{g^{-1}} \zeta(p)$ (so $\mathcal{G}$ acts on itself by an adjoint action), then $C(P, \mathcal{G})$ is naturally anti-isomorphic to the group of gauge transformations $G A(P)$. That is, for $f, f^{\prime} \in G A(P)$ and $\zeta, \zeta^{\prime} \in C(P, \mathcal{G})$ we have that $\left(f \circ f^{\prime}\right)(p)=p\left(\zeta^{\prime}(p) \zeta(p)\right)$.

From the above, it can be readily shown that

$$
f_{*}\left(\sigma_{u *} \mathbf{X}\right)=\left.\frac{d}{d t}\left(R_{\zeta(p)^{-1} \circ \zeta\left(\sigma_{u}(\gamma(t))\right)} f(p)\right)\right|_{t=0}+R_{\zeta(p) *}\left(\sigma_{u *} \mathbf{X}\right),
$$

where $\mathbf{X} \in T \mathcal{M}$ or, writing $\zeta(p)^{-1} \circ \zeta\left(\sigma_{u}(\gamma(t))\right):=e^{t \mathfrak{b}}$ as an element of a one-parameter subgroup of $\mathfrak{G}$,

$$
f_{*}\left(\sigma_{u *} \mathbf{X}\right)=\mathfrak{b}_{f(p)}^{*}+R_{\zeta(p) *}\left(\sigma_{u *} \mathbf{X}\right),
$$

where $\mathfrak{b}_{f(p)}^{*}$ is the fundamental vector field on $f(p)$ corresponding to

$$
\mathfrak{b}=L_{\zeta(p) *}^{-1} \zeta_{*}\left(\sigma_{u} * \mathbf{X}\right) .
$$

Consequently,

$$
\left(\sigma_{u}^{*} f^{*} \omega\right)(\mathbf{X})=\mathfrak{b}+A d_{\left(\sigma_{u}^{*} \zeta\right)(X)^{-1}}\left(\sigma_{u}^{*} \omega\right)(\mathbf{X}) .
$$

In the above expressions, $\omega_{f(p)}$ is a connection 1-form at $f(p) \in P,\left(f^{*} \omega\right)_{p}$ is its pull-back to $p$ with the gauge map $f$ and $\left(\sigma_{u}^{*} f^{*} \omega\right)_{\pi(p)}$ is in turn its pull-back with the local section $\sigma_{u}$ to a 1 -form on $\mathcal{U} \subset \mathcal{M}$, the map $\gamma: \mathbb{R} \rightarrow \mathcal{U}$ is a curve in the base manifold with $\left.\frac{d}{d t} \gamma(t)\right|_{t=0}=\mathbf{X}$, and $\left(\sigma_{u}^{*} \zeta\right)\left(X^{\mu}\right)$ is a space-time-valued element of $\mathcal{G}$.

Write now $\zeta$ as an element of a one-parameter subgroup of $C(P, \mathcal{G})$ by means of the exponential map

$$
\zeta=\exp \left(-t \alpha^{B} T_{B}\right)
$$

where $\alpha^{B} T_{B}:=\boldsymbol{\alpha}$ is an element of the gauge algebra space $C(P, \mathfrak{g})$, and the $T_{B}$ denote the basis matrices of the Lie algebra $\mathfrak{g}$ associated with $\mathcal{G}$. Replacing (4.3) into (4.1) and (4.2) we get

$$
\begin{aligned}
\left(\sigma_{u}^{*}\left(R_{\exp \left(-t \alpha^{B}(p) T_{B}\right)}\right)^{*} \omega\right)(\mathbf{X})= & \left.\frac{d}{d s}\left[\exp \left(t \bar{\alpha}^{B}(X) T_{B}\right) \exp \left(-s \bar{\alpha}^{B}(\gamma(s)) T_{B}\right)\right]\right|_{s=0} \\
& +\operatorname{Ad}_{\exp \left(t \bar{\alpha}^{B}(X) T_{B}\right)}\left(\sigma_{u}^{*} \omega\right)(\mathbf{X})
\end{aligned}
$$

where $\bar{\alpha}^{B}:=\left(\sigma_{u}^{*} \alpha^{B}\right)$. The infinitesimal version of (4.4) follows directly by differentiating both sides of the above equation with respect to the parameter $t$ and evaluating at zero. We therefore arrive at

$$
\delta_{\bar{\alpha}} A:=\left.\frac{d}{d t}\left(\sigma_{u}^{*}\left(R_{\exp \left(-t \alpha^{B} T_{B}\right)}\right)^{*} \omega\right)\right|_{t=0}=-d \overline{\boldsymbol{\alpha}}-[A, \overline{\boldsymbol{\alpha}}]=-D \overline{\boldsymbol{\alpha}} \in \bar{\Lambda}^{1}(\mathcal{M}, \mathfrak{g}),
$$

where $\Lambda^{1}(\mathcal{M}, \mathfrak{g})$ denotes the space of 1 -forms on $\mathcal{M}$ valued in the Lie algebra $\mathfrak{g}$.

Making use of (4.5) in the expression for the Yang-Mills curvature:

$$
F:=D A=d A+\frac{1}{2}[A, A],
$$


we obtain that

$$
\delta_{\bar{\alpha}} F=[\overline{\boldsymbol{\alpha}}, F] .
$$

In the particular case where the one-parameter group is Abelian, it immediately follows that (4.5) and (4.6) simplify to

$$
\delta_{\bar{\alpha}} A=-i d \bar{\alpha}
$$

and

$$
\delta_{\bar{\alpha}} F=0 .
$$

This last result merely states the well know fact that the electromagnetic field strength is gauge independent (i.e. it is independent of the choice of local trivialization).

Moreover, since (4.7) implies that $\delta_{\bar{\alpha}} A_{\mu}=-i \partial_{\mu} \bar{\alpha}$, we obtain, by projecting on the sheet $\Sigma$ with $X^{\mu}{ }_{a}$,

$$
\delta_{\bar{\alpha}} A_{a}=-i \partial_{a} \bar{\alpha}(X(\boldsymbol{\sigma})) .
$$

Let us now turn to the Gauss constraint $G(\boldsymbol{\sigma})$, introduced in (3.4), and to the smearing map

$$
\bar{\alpha} \rightarrow G_{\tau[\bar{\alpha}]}=\int_{\Sigma} d \boldsymbol{\sigma}^{\prime} \bar{\alpha}\left(X\left(\boldsymbol{\sigma}^{\prime}\right)\right) G\left(\boldsymbol{\sigma}^{\prime}\right) .
$$

Comparing (3.16) with (4.8) we see that

$$
i\left\{A_{a}, G_{\tau}[\bar{\alpha}]\right\} \cong \delta_{\bar{\alpha}} A_{a},
$$

so the Poisson bracket of the projection $A_{a}$ of the gauge 4-vector on the space-like hypersurface $\Sigma$ with the Gauss constraint smeared with the scalar function $\bar{\alpha}\left(X\left(\boldsymbol{\sigma}^{\prime}\right)\right)$ is the same as the pullback to $\mathcal{M}$ of the infinitesimal action of the gauge algebra of the PFB with group $U(1)$ on the connection one-form $\omega$ (c.f. equation (4.5)) evaluated on a tangent vector to $\Sigma$.

In addition, for $f \in G A(P)$, it is a simple matter to show that if $\omega$ is a connection 1-form then the pullback $f^{*} \omega$ is also a connection 1 -form. This theorem follows immediately by noting first that the action of $f^{*} \omega$ on a fundamental vector yields its corresponding Lie algebra generator, and second that the requirement $\omega_{p g}\left(R_{g *} X\right)=\operatorname{Ad}_{g^{-1}} \omega_{p}(X)$ in the definition of a connection 1-form is directly satisfied when acting on $\omega$ with the pullback of $f \circ R_{g}=R_{g} \circ f$, which in turn is equivalent the automorphism condition $f(p g)=f(p) g$.

Let now $V$ be a vector space on which $\mathcal{G}$ acts from the left. If $L_{g}: V \rightarrow V$ is linear, then the homomorphism $\mathcal{G} \rightarrow \mathcal{G} L(V)$ by $g \mapsto L_{g}$ is a representation of $\mathcal{G}$. In this case $C(P, V)$ will denote the space of all maps $\zeta: P \rightarrow V$ such that $\zeta(p g)=g^{-1} \cdot \tau(p)$ and the elements of $C(P, V)$ correspond to particle fields.

In particular, $C(P, V)=\bar{\Lambda}^{0}(P, V)$, where, in general, $\bar{\Lambda}^{k}(P, V)$ is the space of $V$-valued differential $k$-forms $\varphi$ on $P$ such that

$$
\begin{aligned}
& R_{g}^{*} \varphi=g^{-1} \cdot \varphi, \\
& \varphi\left(\mathbf{Y}_{1}, \ldots, \mathbf{Y}_{k}\right)=0, \quad \text { if any one of the } \mathbf{Y}_{1}, \ldots \mathbf{Y}_{k} \in T_{p} P \text { is vertical. }
\end{aligned}
$$

Making now use of the exponential map (4.3) it readily follows that

$$
f^{*} \varphi=\zeta^{-1} \cdot \varphi
$$


Or, differentiating with respect to $t$ and evaluating at $t=0$, we arrive at the following infinitesimal version of (4.11):

$$
\delta_{\bar{\alpha}} \bar{\varphi}=\bar{\alpha}^{B} T_{B} \cdot \bar{\varphi} .
$$

Furthermore, related to our discussion in the following sections, note that from the definition of diffeomorphisms we have that $R_{g} \circ f=f \circ R_{g}$, thus acting with the pull-back of this equality on any element $\kappa \in \bar{\Lambda}^{k}(P, V)$, and recalling that the action of the differential $f *$ on a fundamental field $B^{*}$ is a fundamental field, it then immediately follows that $\left(f^{*} \kappa\right)\left(B^{*}\right)=\kappa\left(B^{*}\right)=0$. Hence $f^{*} \kappa \in \Lambda^{k}(P, V), k=0,1,2 \ldots$, and since $C(P, V)=\bar{\Lambda}^{0}(P, V)$ it also follows that the gauge group $G A(P)$ acts on particle fields via pull-back, so that

$$
f^{*} \varphi(p)=\varphi(f(p))
$$

i.e. if $\varphi$ is a particle field, so is also $f^{*} \varphi$.

Using the above results we can now formulate the multiplication rules for gauge and particle fields under gauge transformations, when pulled-back to the base space $\mathcal{M}$. Thus, given two $\mathfrak{g}$-valued potential 1-forms $A, A^{\prime} \in \Lambda^{1}(\mathcal{M}, \mathfrak{g})$, their product is defined by

$$
\left[A, A^{\prime}\right]:=\left(A^{a} \wedge A^{\prime b}\right) \otimes\left[T_{a}, T_{b}\right]
$$

while the product of two particle fields $\varphi_{1}, \varphi_{2} \in C(P, V)$ is by simple point multiplication. Now, as shown previously, the action of an element $f \in G A(P)$ on a connection 1-form and on a particle field is via pull-back (c.f. equations (4.2) and (4.13)) and since the pull-back of a connection is a connection and the pull-back of a particle field is a particle field, it therefore follows that

$$
\begin{aligned}
& f:\left[A, A^{\prime}\right] \leadsto\left[\left(\sigma_{u}^{*} f^{*} \omega_{1}\right),\left(\sigma_{u}^{*} f^{*} \omega_{2}\right)\right], \\
& f:\left(\sigma_{u}^{*} \varphi_{1}\right)(\pi(p)) \cdot\left(\sigma_{u}^{*} \varphi_{2}\right)(\pi(p)) \leadsto\left(\sigma_{u}^{*} f^{*} \varphi_{1}\right)(\pi(p)) \cdot\left(\sigma_{u}^{*} f^{*} \varphi_{2}\right)(\pi(p)) .
\end{aligned}
$$

By (4.5) and (4.12), the infinitesimal expression for the above is:

$$
\begin{aligned}
\delta_{\bar{\alpha}}( & {\left.\left[A, A^{\prime}\right]\left(\mathbf{X}_{1}, \mathbf{X}_{2}\right)\right) } \\
& :=\mu\left[\left(\delta_{\bar{\alpha}} \otimes 1+1 \otimes \delta_{\bar{\alpha}}\right)\left(A^{a}\left(\mathbf{X}_{1}\right) \otimes A^{\prime b}\left(\mathbf{X}_{2}\right)-A^{a}\left(\mathbf{X}_{2}\right) \otimes A^{\prime b}\left(\mathbf{X}_{1}\right)\right)\right] \otimes\left[T_{a}, T_{b}\right] \\
& =\left(\delta_{\bar{\alpha}} A^{a} \wedge A^{\prime b}-A^{a} \wedge \delta_{\bar{\alpha}} A^{\prime b}\right)\left(\mathbf{X}_{1}, \mathbf{X}_{2}\right) \otimes\left[T_{a}, T_{b}\right],
\end{aligned}
$$

and

$$
\delta_{\bar{\alpha}}\left(\bar{\varphi}_{1}(\pi(p)) \cdot \bar{\varphi}_{2}(\pi(p))\right)=\delta_{\bar{\alpha}}\left(\bar{\varphi}_{1}(\pi(p))\right) \cdot \bar{\varphi}_{2}(\pi(p))+\bar{\varphi}_{1}(\pi(p)) \cdot \delta_{\bar{\alpha}}\left(\bar{\varphi}_{2}(\pi(p))\right),
$$

respectively. This last result implies that under an infinitesimal gauge transformation the product of two particle fields transforms according to the Leibniz rule. We can therefore give this infinitesimal transformations the structure of a Hopf algebra with coproduct $\Delta \delta_{\bar{\alpha}}=\delta_{\bar{\alpha}} \otimes 1+1 \otimes \delta_{\bar{\alpha}}$, so that

$$
\delta_{\bar{\alpha}}\left(\bar{\varphi}_{1}(\pi(p)) \cdot \bar{\varphi}_{2}(\pi(p))\right)=\mu\left[\Delta \delta_{\bar{\alpha}}\left(\bar{\varphi}_{1}(\pi(p)) \cdot \bar{\varphi}_{2}(\pi(p))\right)\right] .
$$

From the above discussion we can derive some additional insight into the implications of the PFB point of view of gauge transformations on our previous results. We thus see that since gauge transformations are automorphisms on the fibers that project to the identity on the base space, the Gauss constrain - which we have seen here to be related to the pull-back of the infinitesimal gauge transformations, and which was shown in Section 3 to be needed in order to 
close the algebra in (3.5) - occurs in the extended algebra (3.10) primarily as part of the superHamiltonian and super-momenta associated with the Lie algebra of space-time diffeomorphisms. Its independent appearance is then only as a constraint which, together with $C(\boldsymbol{\sigma}) \simeq 0$, have to be implemented at the end as strong conditions in order to recover the Maxwell theory. This provides an additional natural explanation for why these two constraints can not be mapped into derivations that could lead to an enlarged Lie algebra beyond the one of the space-time diffeomorphisms.

\section{$5 \quad$ Noncommutative gauge theories}

With these results in hand, let us now consider an approach for extending the theory of gauge fields to the noncommutative space-time case, by specifically concentrating on the vacuum Maxwell field discussed in the last two sections, and by following the procedure introduced in [1]. Recall, in particular, that - because of the anti-homomorphism that can be established between the Poisson sub-algebra of the constraints occurring in the first 3 lines of (3.10), for the modified theory in extended phase space, and the Lie algebra $£ \operatorname{diff} \mathcal{M}$ - we can use the latter to investigate the deformed space-time isometries of the system by requiring that this sub-algebra of constraints, modified by the noncommutativity of space-time, should continue obeying the Dirac relations, relative to the Dirac brackets resulting from admitting an arbitrary symplectic structure in the action (3.3). This, as shown in [1], was needed in turn in order to incorporate into the parametrized canonical formalism the dynamical origin of star-noncommutativity from quantum mechanics [14]. Moreover, since the constraints depend on the metric of the embedding space-time, this last step would require in general a well developed theory of quantum mechanics in curved spaces and knowledge of the commutators of the operators representing the phase space coordinates. We shall defer such more general considerations for some future presentation, and concentrate here only on the case of fields on flat Minkowski space-time and the corresponding quantum mechanics for the extended Weyl-Heisenberg group.

Consequently, admitting a symplectic structure in the action (3.8) we have

$$
S[z]=\int d^{4} \sigma\left(\mathcal{B}(z)_{A} \dot{z}^{A}-N^{\alpha}\left({ }^{*} \tilde{\mathcal{H}}_{\alpha}\right)-M G(\boldsymbol{\sigma})-T C(\boldsymbol{\sigma})\right),
$$

with the symplectic variables $z^{A}=\left(X^{\alpha}, A_{a}, \psi ; P_{\alpha}, \pi^{a}, \pi\right)$ and symplectic potentials $\mathcal{B}(z)_{A}$ to be determined by a prescribed symplectic structure. Here $M, T$ are the additional Lagrange multipliers needed to recover Maxwell's electrodynamics and the tildes on the constraints needed of the formerly introduced quantities, in order that their Dirac-bracket algebra originated by the new symplectic structure is identical to their sub-algebra in (3.10). That is, we want to maintain the algebra of these constraints invariant by utilizing new twisted generators. (Observe however, that since the $G(\boldsymbol{\sigma})$ and $C(\boldsymbol{\sigma})$ can not form part of our Lie algebra of space-time isometries, but are strictly constraints to be implemented in order to retrieve Maxwell's electromagnetism, their action on gauge and particle fields will be determined by the arguments given at the end of this section.)

As noted in [1], the symplectic structure is defined by,

$$
\omega_{A B}:=\frac{\partial \mathcal{B}_{B}}{\partial z^{A}}-\frac{\partial \mathcal{B}_{A}}{\partial z^{B}},
$$

from where we can readily solve for the symplectic potentials, which are defined up to a canonical transformation. The resulting second-class constraints can then be eliminated by introducing Dirac brackets, according to a scheme analogous to the one described in the above cited paper, from where the inverse of the symplectic structure is additionally defined through the Diracbrackets for the symplectic variables $z^{A}$. Hence the Dirac brackets for the symplectic variables 
are given by

$$
\left\{z^{A}, z^{B}\right\}^{*}:=\left\{z^{A}, z^{B}\right\}-\left\{z^{A}, \chi_{C}\right\} \omega^{C D}\left\{\chi_{D}, z^{B}\right\}=\omega^{A B},
$$

where $\chi_{A}=\pi_{z^{A}}-\mathcal{B}(z)_{A} \simeq 0$ are the second-class constraints. More specifically, based on the premise that quantum mechanics is a minisuperspace of field theory and for a quantum mechanics on flat Minkowski space-time based on the extended Weyl-Heisenberg group, we have shown in [14] that the WWGM formalism implies that, for the phase space variables to have a dynamical character, we need to modify their algebra by twisting their product according to

$$
\mu\left(X^{\alpha} \otimes X^{\beta}\right) \leadsto \mu_{\theta}\left(X^{\alpha} \otimes X^{\beta}\right):=X^{\alpha}(\tau, \boldsymbol{\sigma}) \star_{\theta} X^{\beta}\left(\tau, \boldsymbol{\sigma}^{\prime}\right)
$$

where

$$
\star_{\theta}:=\exp \left[\frac{i}{2} \theta^{\mu \nu} \int d \sigma^{\prime \prime} \frac{\overleftarrow{\delta}}{\delta X^{\mu}\left(\tau, \boldsymbol{\sigma}^{\prime \prime}\right)} \frac{\vec{\delta}}{\delta X^{\nu}\left(\tau, \boldsymbol{\sigma}^{\prime \prime}\right)}\right]
$$

and where, since the embedding space-time variables are functionals of the foliation, we use functional derivatives. Also, since fields are in turn functions of the embedding space-time variables their multiplication in the noncommutative case is inherited from (5.3). Moreover, using this $\star$-product we can now define the commutator

$$
\begin{aligned}
{\left[X^{\alpha}(\tau, \boldsymbol{\sigma}), X^{\beta}\left(\tau, \boldsymbol{\sigma}^{\prime}\right)\right]_{\theta} } & :=X^{\alpha}(\tau, \boldsymbol{\sigma}) \star_{\theta} X^{\beta}\left(\tau, \boldsymbol{\sigma}^{\prime}\right)-X^{\beta}\left(\tau, \boldsymbol{\sigma}^{\prime}\right) \star_{\theta} X^{\alpha}(\tau, \boldsymbol{\sigma}) \\
& =i \theta^{\alpha \beta} \delta\left(\boldsymbol{\sigma}, \boldsymbol{\sigma}^{\prime}\right),
\end{aligned}
$$

and let

$$
\left\{X^{\alpha}, X^{\beta}\right\}^{*}=\left[X^{\alpha}(\tau, \boldsymbol{\sigma}), X^{\beta}\left(\tau, \boldsymbol{\sigma}^{\prime}\right)\right]_{\star \theta}=i \theta^{\alpha \beta} \delta\left(\boldsymbol{\sigma}, \boldsymbol{\sigma}^{\prime}\right) .
$$

On the other hand, defining the map

$$
\tilde{X}^{\alpha}=X^{\alpha}+\frac{\theta^{\alpha \beta}}{2} P_{\beta},
$$

it follows from (5.2) that

$$
\left\{\tilde{X}^{\alpha}, \tilde{X}^{\beta}\right\}^{*}=0,
$$

and

$$
\left\{{ }^{*} \tilde{\mathcal{H}}_{\alpha}(\vec{\sigma}),{ }^{*} \tilde{\mathcal{H}}_{\beta}\left(\overrightarrow{\sigma^{\prime}}\right)\right\}^{*}=0
$$

Thus, in parallel to (3.7), we have

$$
\left\{{ }^{*} \tilde{H}_{\tau}[\xi],{ }^{*} \tilde{H}_{\tau}[\rho]\right\}^{*}=-{ }^{*} \tilde{H}_{\tau}\left[£_{\xi} \rho\right] .
$$

Furthermore, making the identification $P_{\beta}=-i \frac{\delta}{\delta X^{\beta}}$ in the Darboux map (5.6) we can write

$$
\tilde{X}^{\alpha} \leadsto \hat{\tilde{X}}^{\alpha}=\left(X^{\alpha}\right) \star_{\theta}^{-1}:=\left(X^{\alpha}\right) \exp \left[-\frac{i}{2} \theta^{\mu \nu} \int d \sigma^{\prime \prime} \frac{\overleftarrow{\delta}}{\delta X^{\mu}\left(\tau, \boldsymbol{\sigma}^{\prime \prime}\right)} \frac{\vec{\delta}}{\delta X^{\nu}\left(\tau, \boldsymbol{\sigma}^{\prime \prime}\right)}\right]
$$

where the bi-differential acting from the right on the embedding coordinates $X^{\alpha}$ is the inverse of (5.4). Hence

$$
\left\{\tilde{X}^{\alpha}, \tilde{X}^{\beta}\right\}^{*} \cong\left[\hat{\tilde{X}}^{\alpha}, \hat{\tilde{X}}^{\beta}\right]_{\star_{\theta}}=\left[X^{\alpha}, X^{\beta}\right] \star_{\theta}^{-1}=0,
$$


since under point multiplication the embedding coordinates commute. So the map (5.8) retrieves (5.7).

In addition, since multiplication in the algebra of the operators $\hat{\tilde{X}}^{\alpha}$ is by the $\star_{\theta}$-product we can generalize the last result to

$$
\left\{\left(\tilde{X}^{\alpha}\right)^{m},\left(\tilde{X}^{\beta}\right)^{n}\right\}^{*} \cong\left[\left(\tilde{\tilde{X}}^{\alpha}\right)_{\star}^{m},\left(\hat{\tilde{X}}^{\beta}\right)_{\star}^{n}\right]_{\star_{\theta}}=\left[\left(X^{\alpha}\right)^{m},\left(X^{\beta}\right)^{n}\right] \star_{\theta}^{-1}=0 .
$$

We can therefore conclude from the above that, when replacing the functional dependence on the embedding variables in the constraints in (3.10) by the "tilde" variables (5.6) and the point multiplication of fields by their $\star$-product, the functional form of their algebra is evidently preserved for the noncommutative case. That is,

$$
\left\{{ }^{*} \tilde{H}_{\tau}[\xi],{ }^{*} \tilde{H}_{\tau}[\eta]\right\}^{*} \cong\left[{ }^{*} \hat{H}_{\tau}[\xi],{ }^{*} \hat{H}_{\tau}[\eta]\right]_{\star}{ }^{*}{ }_{\theta}^{-1},
$$

and

$$
{ }^{*} \hat{H}_{\tau}[\xi]=\delta_{\xi} \leadsto{ }^{*} \hat{H}_{\tau}[\xi] \star{ }_{\theta}^{-1}=\delta_{\xi}^{\star},
$$

where the multiplication $\mu_{\theta}$ of the algebra of generators of $\operatorname{diffeomorphisms} \delta_{\xi}^{\star} \in £ \operatorname{diff} \mathcal{M}$ is via the $\star_{\theta}$-product.

Consequently, by using the example of a modified electromagnetism within the context of canonical parametrized field theory, it was shown that, by including additional constraints, Maxwell's equations could be recovered as well as the possibility of also establishing for gauge field theories the anti-homomorphism between Dirac-brackets of the modified constraints and space-time diffeomorphisms. Furthermore using our previous results in [1] where it was shown that noncommutativity in field theory - manifested as the twisting of the algebra of fields has a dynamical origin in the quantum mechanical mini-superspace which, for flat Minkowski space-time, is related to an extended Weyl-Heisenberg group, and including these results into the symplectic structure of the parametrized field theory then allowed us to derive the deformed Lie algebra of the noncommutative space-time diffeomorphisms, as shown by (5.9) and (5.10) above.

Moreover, making use of (5.10) we can summarize the action of space-time diffeomorphisms on particle fields associated with gauge theories, and the transition of the theory to the noncommutative space-time case by means of the following functorial diagrams:

$$
\begin{aligned}
& { }^{*} H_{\tau}[\xi] \in \mathcal{V} \stackrel{\theta}{\mathcal{C}} \mathcal{V}^{\star} \ni^{*} \tilde{H}_{\tau}[\xi]=\int d \vec{\sigma}\left(\tilde{\xi}^{\perp} * \tilde{\mathcal{H}}_{\perp}+\tilde{\xi}^{a}{ }^{*} \tilde{\mathcal{H}}^{a}\right) \\
& { }^{*} \hat{H}_{\tau}[\xi] \in \hat{\mathcal{V}} \stackrel{\mathcal{C}(\theta)}{\longrightarrow} \hat{\mathcal{V}}^{\star} \ni{ }^{*} \hat{H}_{\tau}[\xi] \star_{\theta}^{-1} \equiv \delta_{\xi}^{\star}
\end{aligned}
$$

(where $\mathcal{V}$ denotes the space of constraints satisfying the algebra (3.10), $\mathcal{V}^{\star}$ is the corresponding space of constraints for the space-time noncommutative case with the embedding coordinates mapped according to $(5.6)$ and $\hat{\mathcal{V}}, \hat{\mathcal{V}}^{\star}$ denote the spaces of the Lie algebra of diffeomorphisms and their corresponding twisted form, respectively);

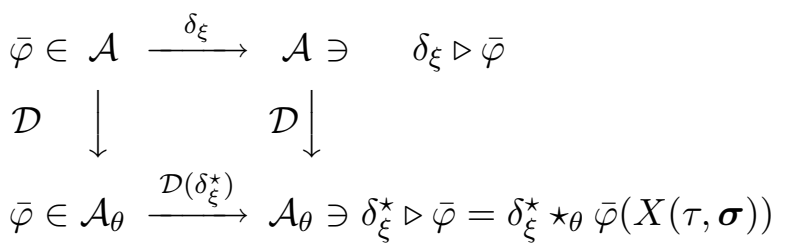

(here $\mathcal{A}$ denotes the module algebra of particle fields $\bar{\varphi} \in C(\mathcal{M}, V)$ with point multiplication $\mu$ and $\mathcal{A}_{\theta}$ is its noncommutative twisting with $\star$-multiplication $\left.\mu_{\theta}:=\mu \circ e^{\frac{i}{2} \theta^{\mu \nu} \partial_{\mu} \otimes \partial_{\nu}}\right)$. 
It then follows from these two diagrams that

$$
\left\{\bar{\varphi},{ }^{*} \hat{H}_{\tau}[\xi]\right\} \cong \delta_{\xi} \triangleright \bar{\varphi} \mapsto \delta_{\xi}^{\star}{ }^{\star} \theta \bar{\varphi}(X(\tau, \boldsymbol{\sigma}))={ }^{*} \hat{H}_{\tau}[\xi] \triangleright \bar{\varphi} .
$$

Note that the diagrams (5.11), (5.12) and equation (5.13) provide an explicit expression for the mappings $\delta_{\rho} \mapsto \delta_{\rho}^{\star}$, which in turn imply

$$
\left[\delta_{\rho}^{\star}, \delta_{\eta}^{\star}\right]_{\star \theta}=\delta_{£_{\rho} \eta}^{\star},
$$

and

$$
\delta_{\rho}^{\star} \star_{\theta}\left(\bar{\varphi}_{1} \star_{\theta} \bar{\varphi}_{2}\right)=\delta_{\rho}\left(\bar{\varphi}_{1} \star_{\theta} \bar{\varphi}_{2}\right),
$$

where $\bar{\varphi}_{1}, \bar{\varphi}_{2} \in \mathcal{A}_{\theta}$.

Note also that the universal envelopes $U(\hat{\mathcal{V}})$ and $U\left(\hat{\mathcal{V}}^{\star}\right)$ of the derivations $\delta_{\xi}$ and twisted derivations $\delta_{\xi}^{\star}$ can be given the structure of Hopf algebras. Thus, in particular, we can obtain an explicit expression for the coproduct in $U\left(\hat{\mathcal{V}}^{\star}\right)$ by making use of the duality between product and coproduct, followed by the application of equation (5.14). We get

$$
\begin{aligned}
& \mu_{\theta} \circ \Delta\left(\delta_{\rho}^{\star}\right)\left(\bar{\varphi}_{1} \otimes \bar{\varphi}_{2}\right)=\delta_{\rho}^{\star} \star_{\theta}\left(\bar{\varphi}_{1} \star_{\theta} \bar{\varphi}_{2}\right)=\delta_{\rho}\left(\bar{\varphi}_{1} \star_{\theta} \bar{\varphi}_{2}\right) \\
& =\mu\left(\delta_{\rho} \otimes 1+1 \otimes \delta_{\rho}\right)\left(e^{\frac{i}{2} \theta^{\mu \nu} \partial_{\mu} \otimes \partial_{\nu}} \bar{\varphi}_{1} \otimes \bar{\varphi}_{2}\right) \\
& =\sum_{n} \frac{1}{n !}\left(\frac{i}{2}\right)^{n} \theta^{\mu_{1} \nu_{1}} \cdots \theta^{\mu_{n} \nu_{n}}\left[\left(\delta_{\rho}^{\star} \star_{\theta} \partial_{\mu_{1} \ldots \mu_{n}} \bar{\varphi}_{1}\right) e^{-\frac{i}{2} \theta^{\mu \nu} \overleftarrow{\partial}_{\mu} \vec{\partial}_{\nu} \star_{\theta} \partial_{\nu_{1} \ldots \nu_{n}} \bar{\varphi}_{2}}\right.
\end{aligned}
$$

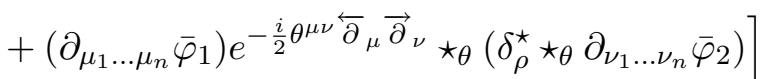

$$
\begin{aligned}
& =\mu_{\theta} \circ\left[e^{-\frac{i}{2} \theta^{\mu \nu} \partial_{\mu} \otimes \partial_{\nu}}\left(\delta_{\rho}^{\star} \otimes 1+1 \otimes \delta_{\rho}^{\star}\right) e^{\frac{i}{2} \theta^{\mu \nu} \partial_{\mu} \otimes \partial_{\nu}}\right]\left(\bar{\varphi}_{1} \otimes \bar{\varphi}_{2}\right) \text {. }
\end{aligned}
$$

This result compares with the Leibniz rule given in [6]. Furthermore, if we let $\mathcal{F}=e^{-\frac{i}{2} \theta^{\mu \nu} \partial_{\mu} \otimes \partial_{\nu}} \in$ $U(\hat{\mathcal{V}}) \otimes U(\hat{\mathcal{V}})$, and define $\bar{\varphi}_{1} \star_{\theta} \bar{\varphi}_{2}=\mu_{\theta}\left(\bar{\varphi}_{1} \otimes \bar{\varphi}_{2}\right):=\mu\left(\mathcal{F}^{-1} \triangleright\left(\bar{\varphi}_{1} \otimes \bar{\varphi}_{2}\right)\right)$, we then have [22, 23]:

$$
\begin{aligned}
\delta_{\rho}\left(\bar{\varphi}_{1} \star_{\theta} \bar{\varphi}_{2}\right) & \left.=\delta_{\rho} \triangleright \mu\left(\mathcal{F}^{-1} \triangleright\left(\bar{\varphi}_{1} \otimes \bar{\varphi}_{2}\right)\right)=\mu\left[\left(\Delta \delta_{\rho}\right) \mathcal{F}^{-1} \triangleright\left(\bar{\varphi}_{1} \otimes \bar{\varphi}_{2}\right)\right)\right] \\
& \left.=\mu \mathcal{F}^{-1}\left[\left(\mathcal{F}\left(\Delta \delta_{\rho}\right) \mathcal{F}^{-1}\right)\left(\left(\bar{\varphi}_{1} \otimes \bar{\varphi}_{2}\right)\right)\right)\right] \\
& \left.=\mu_{\theta}\left[\left(\mathcal{F}\left(\Delta \delta_{\rho}\right) \mathcal{F}^{-1}\right)\left(\left(\bar{\varphi}_{1} \otimes \bar{\varphi}_{2}\right)\right)\right)\right] .
\end{aligned}
$$

Thus, the undeformed coproduct of the symmetry Hopf algebra $U(\hat{\mathcal{V}})$ is related to the Drinfeld twist $\Delta^{\mathcal{F}}$ by the inner endomorphism $\Delta^{\mathcal{F}} \delta_{\rho}:=\left(\mathcal{F}\left(\Delta \delta_{\rho}\right) \mathcal{F}^{-1}\right)$ and, by virtue of (5.15), it preserves the covariance:

$$
\begin{aligned}
\left.\delta_{\rho} \triangleright\left(\left(\bar{\varphi}_{1} \cdot \bar{\varphi}_{2}\right)\right)\right) & \left.=\mu \circ\left[\Delta\left(\delta_{\rho}\right)\left(\bar{\varphi}_{1} \otimes \bar{\varphi}_{2}\right)\right)\right]=\left(\delta_{\rho(1)} \triangleright \bar{\varphi}_{1}\right) \cdot\left(\delta_{\rho(2)} \triangleright \bar{\varphi}_{2}\right) \\
& \left.\stackrel{\theta}{\rightarrow} \delta_{\rho}^{\star} \triangleright\left(\bar{\varphi}_{1} \star_{\theta} \bar{\varphi}_{2}\right)=\left(\delta_{\rho(1)}^{\star} \triangleright \bar{\varphi}_{1}\right) \star_{\theta}\left(\delta_{\rho(2)}^{\star}\right) \triangleright \bar{\varphi}_{2}\right),
\end{aligned}
$$

where we have used the Sweedler notation for the coproduct. Consequently, the twisting of the coproduct is tied to the deformation $\mu \rightarrow \mu_{\theta}$ of the product when the last one is defined by

$$
\bar{\varphi}_{1} \star_{\theta} \bar{\varphi}_{2}:=\left(\mathcal{F}_{(1)}^{-1} \triangleright \bar{\varphi}_{1}\right)\left(\mathcal{F}_{(2)}^{-1} \triangleright \bar{\varphi}_{2}\right) .
$$

We want to reiterate at this point that the $\star$-product, associated with the algebra $\mathcal{A}_{\theta}$, that we have been considering here is the one originated when considering in turn the flat-Minkowski space-time quantum mechanics generated by the extended Weyl-Heisenberg group $H_{5}$, for the even more particular case of an extension of the Lie algebra of $H_{5}$ by the commutator $\left[X^{\mu}, X^{\nu}\right]=$ 
$i \theta^{\mu \nu}$, for the simplest case when $\theta^{\mu \nu}=$ const. In this case the generators $\delta_{\rho}$ of isometries become the infinitesimal generators of the Poincaré group of transformations, and the coproduct defined in this equation reduces to the twisted coproduct considered by e.g. [24] (see also e.g. [5] and $[25,26])$. Since the embedding coordinates in the canonical parametrized theory can in general be associated to a curved space-time manifold and, since the constraints and related diffeomorphisms are constructed for such spaces, it seems possible in principle that our formalism could be extended to curved space-time backgrounds with a $\star$-product determined by the Lie algebra associated with, for instance, a given homogeneous space. This would imply finding first the equivalent of the mapping (5.6) and also, of course, the realization of this map in terms of the *-product, perhaps by a procedure based on the deformation quantization formalism developed by Stratonovich [27]. A fairly simple example of the above is the Darboux map given in [29], for the case of the Snyder algebra [28]. However, finding a full realization of the $\star$-product is a more difficult job.

In equation (4.15) of the previous section we derived the expression for the infinitesimal gauge transformation on a product of particle fields in $\mathcal{A}$. Let us now consider the effect of such a gauge transformation on the product of two particle fields in $\mathcal{A}_{\theta}$ when we have spacetime noncommutativity. For this purpose we first recall equation (4.13) which shows that if $\varphi$ is a particle field, so is its gauge transformation by pull-back, i.e. $\varphi \in C(P, V) \Rightarrow \varphi^{\prime}:=$ $f^{*} \varphi \in C(P, V)$. From this it follows that to a given element of $C(P, V)$ we can always associate another one which is the pull-back of the former, thus the twisted product of the pull-back with the section $\sigma_{u}$ of any pair of particle fields can be written as

$$
\bar{\varphi}_{1}^{\prime} \star_{\theta} \bar{\varphi}_{2}^{\prime}=\left(\sigma_{u}^{*}\left(f^{*} \varphi_{1}\right)\right) \star_{\theta}\left(\sigma_{u}^{*}\left(f^{*} \varphi_{2}\right)\right) .
$$

Observe however that, because of the noncommutativity that the algebra (5.5) of the embedding coordinates is required to satisfy, the pull-back to $\mathcal{M}$ of the gauge transformation (4.11) now should be understood as $\sigma_{u}^{*} f^{*} \varphi=\bar{\zeta}_{\star}^{-1}(X) \star_{\theta} \bar{\varphi}(X)$; so that

$$
\bar{\varphi}_{1}^{\prime} \star_{\theta} \bar{\varphi}_{2}^{\prime}=\left(\bar{\zeta}_{\star}^{-1} \star_{\theta} \bar{\varphi}_{1}\right) \star_{\theta}\left(\bar{\zeta}_{\star}^{-1} \star_{\theta} \bar{\varphi}_{2}\right),
$$

where, due to the noncommutativity, equation (4.3) is replaced by

$$
\bar{\zeta}^{-1} \leadsto \bar{\zeta}_{\star}^{-1}=\exp _{\star}(t \overline{\boldsymbol{\alpha}}(X)):=1+t \overline{\boldsymbol{\alpha}}+\frac{t^{2}}{2} \overline{\boldsymbol{\alpha}} \star_{\theta} \overline{\boldsymbol{\alpha}}+\cdots
$$

Using the infinitesimal version of this map we have that $\bar{\varphi}_{1}^{\prime}=\bar{\varphi}+\overline{\boldsymbol{\alpha}} \star_{\theta} \bar{\varphi}$, so that (5.16) becomes

$$
\delta_{\bar{\alpha}}:\left(\bar{\varphi}_{1} \star_{\theta} \bar{\varphi}_{2}\right):=\bar{\varphi}_{1}^{\prime} \star_{\theta} \bar{\varphi}_{2}^{\prime}=\left(\overline{\boldsymbol{\alpha}}(X) \star_{\theta} \bar{\varphi}_{1}(X)\right) \star_{\theta} \bar{\varphi}_{2}+\bar{\varphi}_{1} \star_{\theta}\left(\overline{\boldsymbol{\alpha}}(X) \star_{\theta} \bar{\varphi}_{2}(X)\right) .
$$

By a similar argument, since $f \in G A(P)$ also maps connections into connections, its infinitesimal action on the $\star$-product of two gauge fields (c.f. (4.14)) goes into

$$
\begin{aligned}
\delta_{\bar{\alpha}}:( & {\left.\left[A, A^{\prime}\right]_{\star_{\theta}}\left(\mathbf{X}_{1}, \mathbf{X}_{2}\right)\right):=-\left[\left(d \bar{\alpha}^{A}\left(\mathbf{X}_{1}\right)+\frac{1}{2} c^{A}{ }_{C D}\left[A^{C}\left(\mathbf{X}_{1}\right), \bar{\alpha}^{D}\left(\mathbf{X}_{1}\right)\right]_{\star_{\theta}}\right) \star_{\theta} A^{B}\left(\mathbf{X}_{2}\right)\right.} \\
& -\left(d \bar{\alpha}^{A}\left(\mathbf{X}_{2}\right)+\frac{1}{2} c^{A}{ }_{C D}\left[A^{C}\left(\mathbf{X}_{2}\right), \bar{\alpha}^{D}\left(\mathbf{X}_{2}\right)\right]_{\star_{\theta}}\right) \star_{\theta} A^{B}\left(\mathbf{X}_{1}\right) \\
& +A^{A}\left(\mathbf{X}_{1}\right) \star_{\theta}\left(d \bar{\alpha}^{B}\left(\mathbf{X}_{2}\right)+\frac{1}{2} c^{B}{ }_{C D}\left[A^{\prime C}\left(\mathbf{X}_{2}\right), \bar{\alpha}^{D}\left(\mathbf{X}_{2}\right)\right]_{\star_{\theta}}\right) \\
& \left.-A^{A}\left(\mathbf{X}_{2}\right) \star_{\theta}\left(d \bar{\alpha}^{B}\left(\mathbf{X}_{1}\right)+\frac{1}{2} c^{B}{ }_{C D}\left[A^{\prime C}\left(\mathbf{X}_{1}\right), \bar{\alpha}^{D}\left(\mathbf{X}_{1}\right)\right]_{\star_{\theta}}\right)\right] \otimes\left[T_{A}, T_{B}\right] .
\end{aligned}
$$

Note that we have written the last two equations for the general case of any group of gauge transformations, where $\overline{\boldsymbol{\alpha}}(X)=\bar{\alpha}^{B} T_{B}$, in order to underline the fact that, because of the $\star-$ product in the multiplication of the fields one needs to apply the constraint that these $\mathrm{NC}$ gauge 
groups have to be in the fundamental or adjoint unitary representation (i.e. $T_{A} \in U(n)$ ), since only in this representation the gauge group closes (c.f. e.g. [12, 19]). See however also [20] for arguments tending to circumvent this constraint). Hence, in the $\mathrm{NC}$ case the generators of gauge symmetry act on particle fields with the fundamental representation

$$
\bar{\varphi} \leadsto \bar{\varphi}^{\prime}=\zeta_{\star}^{-1} \star_{\theta} \bar{\varphi}=\exp _{\star}(t \overline{\boldsymbol{\alpha}}(X)) \star_{\theta} \bar{\varphi},
$$

while on gauge fields the action is via the adjoint representation

$$
A(\mathbf{X}) \leadsto A^{\prime}(\mathbf{X})=\zeta_{\star}^{-1} \star_{\theta} A(\mathbf{X}) \star_{\theta} \zeta_{\star}+\zeta_{\star}^{-1} \star_{\theta}\left(d \zeta_{\star}\right)(\mathbf{X})
$$

Equations (5.18) and (5.19) agree with those on which [11] is based when remarking on some of the conclusions on deformed gauge theories arrived at in $[10,9,30,31]$. Indeed, one basic idea in this other approach of gauge twisted theories is the assumption that the gauge generators $\delta_{\bar{\alpha}}:=\overline{\boldsymbol{\alpha}}(X)=\bar{\alpha}^{B}(X) T_{B}$ act on particle and gauge fields with the usual point product, so instead of (5.17) they define

$$
\delta_{\bar{\alpha}}\left(\bar{\varphi}_{1} \star_{\theta} \bar{\varphi}_{2}\right):=\left(\delta_{\bar{\alpha}} \bar{\varphi}_{1}\right) \star_{\theta} \bar{\varphi}_{2}+\bar{\varphi}_{1} \star_{\theta}\left(\delta_{\bar{\alpha}} \bar{\varphi}_{2}\right) .
$$

Moreover, by assuming that the algebra of the gauge generators can be given an additional Hopf bialgebra structure, and that the derivatives of any order of the gauge and particle fields are, as noted in [11], in the same representation of the gauge algebra as the fields themselves, one could further write

$$
\begin{aligned}
\delta_{\bar{\alpha}}\left(\bar{\varphi}_{1} \star_{\theta} \bar{\varphi}_{2}\right) & =\left(\overline{\boldsymbol{\alpha}}(X) \bar{\varphi}_{1}\right) \star_{\theta} \bar{\varphi}_{2}+\bar{\varphi}_{1} \star_{\theta} \overline{\boldsymbol{\alpha}}(X) \bar{\varphi}_{2} . \\
& =\mu \circ\left(\delta_{\bar{\alpha}} \otimes 1+1 \otimes \delta_{\bar{\alpha}}\right) \circ\left(e^{\frac{i}{2} \theta^{\mu \nu} \partial_{\mu} \otimes \partial_{\nu}} \bar{\varphi}_{1} \otimes \bar{\varphi}_{2}\right) \\
& =\mu_{\theta}\left[\left(\Delta^{\mathcal{F}} \delta_{\bar{\alpha}}\right) \circ\left(\bar{\varphi}_{1} \otimes \bar{\varphi}_{2}\right)\right] .
\end{aligned}
$$

Assuming a scalar particle field for simplicity and setting $\bar{\varphi}_{2}=\partial_{\mu} \bar{\varphi}$ and $\bar{\varphi}_{1}=\partial_{\mu} \bar{\varphi}^{\dagger}$, it can be readily seen that one immediate consequence of the extra assumption leading to equating the last two lines in (5.21) with the first one is that the latter then yields:

$$
\delta_{\bar{\alpha}}\left(\partial_{\mu} \bar{\varphi}^{\dagger} \star_{\theta} \partial_{\mu} \bar{\varphi}\right)=0,
$$

which implies that the kinetic terms in the Lagrangian of the particle fields are invariant by themselves, so there would be no need to introduce the gauge potentials to achieve gauge invariance of the theory. Consequently, since (5.21) only fully agrees with (5.17) when $\overline{\boldsymbol{\alpha}}$ is coordinate independent, there appears to be a discrepancy as a consequence of local internal symmetry between assuming the validity of (5.20) and some essential aspects of the theory of gauge invariance.

Recall furthermore, that a Drinfeld twist (c.f. e.g. [22, 23, 32]) involves a simultaneous and covariant deformation of the product of an algebra $\mathcal{A}$ of functions and the coproduct of a bialgebra $H$. More specifically, the algebra $\mathcal{A}$ is a module algebra $(H$-module algebra) over a Hopf bialgebra whose elements are in the universal enveloping algebra $U(L)$ of a Lie algebra $L$, such that if $x \in L$ then $\Delta(x)=x \otimes 1+1 \otimes x$, and $x(a b)=x(a) b+a x(b) \forall a, b \in \mathcal{A}$, so that $x$ acts as a derivation. On the other hand, as shown by equations (4.9) and (4.10), the infinitesimal gauge transformation of the gauge potential is given by the Poisson bracket of the smeared Gauss constraint $G_{\tau}[\bar{\alpha}]$ with the gauge potential; but, as it was also shown in Section 3 of this paper, the $\delta_{\bar{\alpha}}$ can not be made isomorphic to a derivation operator acting as such on the gauge potentials or particle fields, contrary to the case of the smeared super-Hamiltonian and super-momenta constraints. Consequently the algebra of the infinitesimal gauge transformations can not be considered as part of the Hopf algebra of the space-time diffeomorfisms $\delta_{\xi}$, associated with Lie 
algebra $L$ and its universal envelope, from which a Drinfeld twist could be properly constructed. Note also that in the context of the canonical parametrized formalism, the Gauss constraint is defined on the spacelike hypersurface $\Sigma$ and, again contrary to the super-Hamiltonian and super-momenta constraints, does not depend on the embedding variables. This translates in the fact that for the $\mathrm{NC}$ case the space-time diffeomorphisms $\delta_{\xi}$, on the one hand, and the infinitesimal gauge transformations $\delta_{\bar{\alpha}}$, on the other, act quite differently on the gauge and particle fields. This is clearly seen when comparing the actions (5.10) and (5.18) on the gauge and particle fields, as well as their actions (5.15) and (5.17) on their respective products.

It thus appears from our present results as well as from those in [1] (where the noncommutative reparametrized scalar field was considered and its respective constraints together with their anti-homomorphic relation to space-time diffeomorphisms was explicitly established), that it might not be possible to extend the concept of a Drinfeld twist symmetry to include gauge symmetries, when considering the minimal coupling of gauge and particle fields in order to investigate a full model of $\mathrm{NC}$ theory in the context of the canonical reparametrized theory (see e.g. [12] regarding this point).

However, if one were to consider relaxing the concept of twisted symmetries and modify the definition of a deformed Leibniz rule (such as the one exhibited in (5.20)), several different twists and gauge invariants may be constructed that would lead to alternate formulations for NC gauge theories. Some new ideas in this context that might help to remove some of the inconsistencies pointed out here as well as elsewhere, are discussed in [33, 34]. This would involve, essentially, assuming different deformations of products of elements in the same algebra of space-time functions $\mathcal{A}$, when considering different transformation groups. Such an assumption however, would be hard to reconcile with the point of view that the product in this algebra of functions is inherited from the deformation of the algebra of space-time coordinates and its dynamical origin in the quantum mechanical mini-superspace.

As it was remarked previously the $\star$-product considered so far applies to an underlying flat Minkowski space-time, and the corresponding twisted isometries refer then to the Poincaré group. It is interesting to observe, however, that our formalism admits a natural extension of (5.4) which allows us to consider much more general symplectic structures than (5.1) that would imply noncommutativity among all the symplectic variables $z^{A}=\left(X^{\alpha}, A_{a}, \psi ; P_{\alpha}, \pi^{a}, \pi\right)$. Moreover, because of the appearance of the embedding metric in the canonical parametrized formalism, this could lead in turn to the possibility of extending our analysis to the case of twisted isometries on curved space backgrounds.

Even within the flat Minkowski space-time case, we could have a more general symplectic structure that would lead to a different $\star$-product with bi-differentials involving some of the other fields in the theory. Consider for instance the symplectic structure resulting in the Dirac brackets:

$$
\begin{aligned}
& \left\{X^{\alpha}, X^{\beta}\right\}^{*}=i \theta^{\alpha \beta}, \quad\left\{X^{\alpha}, P_{\beta}\right\}^{*}=i \delta_{\alpha}^{\beta}, \quad\left\{P_{\alpha}, P_{\beta}\right\}^{*}=0, \\
& \left\{A_{a}, A_{b}\right\}^{*}=0, \quad\left\{A_{a}, \pi^{b}\right\}^{*}=i \delta_{a}^{b}, \quad\left\{\pi^{a}, \pi^{b}\right\}^{*}=i \beta^{a b},
\end{aligned}
$$

(and the remainder equal to zero). Here the Darboux map, that takes us from the extended algebra (5.22) to the usual Heisenberg algebra, is given by the transformations:

$$
\tilde{X}^{\alpha}=X^{\alpha}+\frac{\theta^{\alpha \beta}}{2} P_{\beta}, \quad \tilde{\pi}^{a}=\pi^{a}+\frac{\beta^{a b}}{2} A_{b} .
$$

These maps are unique up to a canonical transformation on the phase-space $\left(X^{\alpha}, P_{\alpha}, A_{a}, \pi^{a}\right)$. In order to construct the deformed constraints, note that in the expressions for $\Phi_{0}$ and $\Phi_{a}$ in (3.4) there appear the projectors $n^{\alpha}(\sigma, X)$ and $X_{a}^{\alpha}(\sigma, X)$ as well as the 3-metric $\gamma_{a b}$, all of which are functionals of the space-time embedding coordinates $X^{\alpha}$. These quantities thus need 
to be modified according to (5.23). On the other hand, the Gauss constraint also requires to be modified in order that the Dirac bracket algebra of the new constraints be the same as the Poisson algebra of the original ones. The resulting deformed constraints are then:

$$
\begin{aligned}
& \tilde{\Phi}_{0}=P_{\alpha} \tilde{n}^{\alpha}+\frac{1}{2} \tilde{\gamma}^{-1 / 2} \tilde{\gamma}_{a b} \tilde{\pi}^{a} \tilde{\pi}^{b}+\frac{1}{4} \tilde{\gamma}^{1 / 2} \tilde{\gamma}^{a c} \tilde{\gamma}^{b d} F_{a b} F_{c d}, \\
& \tilde{\Phi}_{a}=P_{\alpha} \tilde{X}_{, a}^{\alpha}-F_{a b} \tilde{\pi}^{b}, \quad \tilde{G}=\tilde{\pi}_{, a}^{a},
\end{aligned}
$$

where the tilde on top of a symbol denotes the replacement of the space-time coordinates according to (5.23). However, one point to observe is even that the constraints have been deformed by the fact that their algebra involves now Dirac brackets instead of Poisson brackets, the Darboux transformations (5.23) preserve the functional form of their algebra, so they can still be made anti-homomorphic to an algebra of deformed space-time diffeomorphisms, by a procedure analogous to the one described here. Also note, in particular, that the original fields $z^{A}=\left(X^{\alpha}, A_{a}, \psi ; P_{\alpha}, \pi^{a}, \pi\right)$ will now transform according to the twisted diffeomorphisms of the theory. Thus, while the electric field $\pi^{a}$ will no longer be gauge invariant, the new field $\tilde{\pi}^{a}$ will be, under the gauge transformation associated with the modified Gauss constraint. Note also that the last equation in (5.22)implies that the Drinfeld deformation of the algebra of functions of the fields involves a $\star$-product which is a composition of (5.4) with

$$
\star_{\beta}:=\exp \left[\frac{i}{2} \beta^{a b} \int d \sigma^{\prime \prime} \frac{\overleftarrow{\delta}}{\delta \pi^{a}\left(\tau, \boldsymbol{\sigma}^{\prime \prime}\right)} \frac{\vec{\delta}}{\delta \pi^{b}\left(\tau, \boldsymbol{\sigma}^{\prime \prime}\right)}\right]
$$

\section{Acknowledgements}

The authors are grateful to Prof. Karel Kuchař for fruitful discussions and clarifications concerning his work on parametrized canonical quantization. They are also grateful to the referees for some very pertinent comments and suggestions which helped to clarify considerably some points in the manuscript. The authors also acknowledge partial support from CONACyT projects UA7899-F (M.R.) and 47211-F (J.D.V.) and DGAPA-UNAM grant IN109107 (J.D.V.).

\section{References}

[1] Rosenbaum M., Vergara J.D., Juarez L.R., Canonical quantization, space-time noncommutativity and deformed symmetries in field theory, J. Phys. A: Math. Theor. 40 (2007), 10367-10382, hep-th/0611160.

[2] Isham C.J., Kuchař K.V., Representations of space-time diffeomorphisms. I. Canonical parametrized field theories, Ann. Physics 164 (1985), 288-315.

[3] Isham C.J., Kuchař K.V., Representations of space-time diffeomorphisms. II. Canonical geometrodynamics, Ann. Physics 164 (1985), 316-333.

[4] Kuchař K.V., Stone S.L., A canonical representation of space-time diffeomorphisms for the parametrized maxwell field, Classical Quantum Gravity 4 (1987), 319-328.

[5] Chaichian M., Kulish P.P., Nishijima K., Tureanu A., On a Lorentz-invariant interpretation of noncommutative space-time and its implications on noncommutative QFT, Phys. Lett. B 604 (2004), 98-102, hep-th/0408069.

[6] Aschieri P., Blohmann C., Dimitrijevic M., Meyer F., Schupp P., Wess J., A gravity theory on noncommutative spaces, Classical Quantum Gravity 22 (2005), 3511-3532, hep-th/0504183.

[7] Aschieri P., Dimitrijevic M., Meyer F., Wess J., Noncommutative geometry and gravity, Classical Quantum Gravity 23 (2006), 1883-1912, hep-th/0510059.

[8] Wess J., Einstein-Riemann gravity on deformed spaces, SIGMA 2 (2006), 089, 9 pages, hep-th/0611025.

[9] Vassilevich D.V., Twist to close, Modern Phys. Lett. A 21 (2006), 1279-1284, hep-th/0602185.

[10] Aschieri P., Dimitrijevic M., Meyer F., Schraml S., Wess J., Twisted gauge theories, Lett. Math. Phys. 78 (2006), 61-71, hep-th/0603024. 
[11] Chaichian M., Tureanu A., Twist symmetry and gauge invariance, Phys. Lett. B 637 (2006), 199-202, hep-th/0604025.

[12] Chaichian M., Tureanu A., Zet G., Twist as a symmetry principle and the noncommutative gauge theory formulation, Phys. Lett. B 651 (2007), 319-323, hep-th/0607179.

[13] Banerjee R., Samanta S., Gauge symmetries on $\theta$-deformed spaces, J. High Energy Phys. 2007 (2007), no. 02, 046, 17 pages, hep-th/0611249.

[14] Rosenbaum M., Vergara J.D., Juárez L.R., Dynamical origin of the $\star_{\theta}$-noncommutativity in field theory from quantum mechanics, Phys. Lett. A 354 (2006), 389-395, hep-th/0604038.

[15] Kuchař K.V., Canonical quantization of gravity, in Relativity, Astrophysics and Cosmology, Editor W. Israel, Reidel Pub. Co., Dordrecht, Holland, 1973, 237-288.

[16] Kuchař K.V., Kinematics of tensor fields in hyperspace. II, J. Math. Phys. 17 (1976), 792-800.

[17] Dirac P.A.M., Lectures on quantum mechanics, Belfast Graduate School of Science, Yeshiva University New York, 1964.

[18] Kuchař K.V., Kinematics of tensor fields in hyperspace. III, J. Math. Phys. 17 (1976), 801-820.

[19] Chaichian M., Presnajder P., Shikh-Jabbari M.M., Tureanu A., Noncommutative gauge field theories: a nogo theorem, Phys. Lett. B 526 (2002), 132-136, hep-th/0107037.

[20] Arai M., Saxell S., Tureanu A., Uekusa N., Circumventing the no-go theorem in noncommutative gauge field theory, Phys. Lett. B 661 (2008), 210-215, arXiv:0710.3513.

[21] Bleecker D., Gauge theory and variational principles, Addison-Wesley Publishing Co., Reading, Mass., 1981.

[22] Blohmann Ch., Realization of $q$ deformed space-time as star product by a Drinfeld twist, in Group 24: Physical and Mathematical Aspects of Symmetries, Proceedings of International Colloquium on Group Theoretical Methods in Physics (July 15-20, 2002, Paris), Editors J.P. Gazeau, R. Kerner, J.P. Antoine, S. Metens and J.Y. Thibon, IOP Conference Series, Vol. 173, 2003, 443-446, math.QA/0402199.

[23] Oeckl R., Untwisting noncommutative $\mathbb{R}^{d}$ and the equivalence of quantum field theories, Nuclear Phys. B 581 (2000), 559-574, hep-th/0003018.

[24] Chaichian M., Presnajder M., Tureanu A., New concept of relativistic invariance in NC space-time: twisted Poincaré symmetry and its implications, Phys. Rev. Lett. 94 (2005), 151602, 15 pages, hep-th/0409096.

[25] Wess J., Deformed coordinate spaces: derivatives, in Proceedings of the BW2003 Workshop on Mathematical, Theoretical and Phenomenological Challenges beyond the Standard Model: Perspectives of Balkans Collaboration (August 29 - September 2, 2003, Vrnjacka Banja, Serbia), Editors G. Djordjevic, L. Nesic and J. Wess, World Scientific, 2005, 122-128, hep-th/0408080.

[26] Kosinski P., Maslanka P., Lorentz-invariant interpretation of noncommutative space-time: global version, hep-th/0408100.

[27] Stratonovich R.L., On distributions in representation space, Zh. Eksp. Teor. Fiz. 31 (1956), 1012-1020 (English transl.: Sov. Phys. JETP 4 (1957), 891-898).

[28] Snyder H.S., Quantized space-time, Phys. Rev. 71 (1947), 38-41.

[29] Romero J.M., Vergara J.D., Santiago J.A., Noncommutative spaces, the quantum of time and the Lorentz symmetry, Phys. Rev. D 75 (2007), 065008, 7 pages, hep-th/0702113.

[30] Wess J., Deformed gauge theories, J. Phys. Conf. Ser. 53 (2006), 752-763, hep-th/0608135.

[31] Giller S., Gonera C., Kosinski P., Maslanka P., On the consistency of twisted gauge theory, Phys. Lett. B 655 (2007), 80-83, hep-th/0701014.

[32] Kassel Ch., Quantum groups, Springer-Verlag, New York - Berlin - Heidelberg, 1995.

[33] Vassilevich D.V., Symmetries in noncommutative field theories: Hopf versus Lie, arXiv:0711.4091.

[34] Duenas-Vidal A., Vazquez-Mozo M.A., Twisted invariances of noncommutative gauge theories, arXiv:0802.4201. 